\title{
Designing choice experiments by optimizing the complexity level to individual abilities
}

\author{
Vishva M. Danthurebandara \\ Faculty of Business and Economics, KU Leuven, Naamsestraat 69, B-3000 Leuven, Belgium. \\ Email: Vishva.Danthurebandara@kuleuven.be \\ Tel: $+32(0) 16326963$ \\ Fax: $+32(0) 16326624$

\section{Martina Vandebroek} \\ Faculty of Business and Economics \& Leuven Statistics Research Centre, KU Leuven, Naamsestraat \\ 69, B-3000 Leuven, Belgium. \\ Email: Martina.Vandebroek@kuleuven.be \\ Tel: $+32(0) 16326975$ \\ Fax: $+32(0) 16326624$
}

\section{Jie Yu}

Faculty of Business and Economics, KU Leuven, Naamsestraat 69, B-3000 Leuven, Belgium.

Email: Jie.Yu@kuleuven.be

Tel: $+32(0) 16326962$

Fax: +32 (0)16 326624 


\title{
Designing choice experiments by optimizing the complexity level to individual abilities *
}

\author{
Vishva M. Danthurebandara ${ }^{\dagger}$ Martina Vandebroek ${ }^{\ddagger}$ and Jie $\mathrm{Yu}^{\S}$
}

\begin{abstract}
It has been proven repeatedly in psychology and behavioural decision theory that the complexity of the choice sets affects the consistency of the responses in choice experiments. A handful of studies can be found in the discrete choice literature that take this dependency explicitly into account at the estimation stage. But there is only limited research that investigates how the choice complexity affects the efficiency of the choice design.

In this research we propose choice designs in order to estimate the heteroscedastic mixed logit model which is parametrized to model the preference heterogeneity as well as the scale heterogeneity due to the choice complexity. The heteroscedastic model assumes that the scale factor is an exponentiated linear function of some complexity measures. An increase in choice complexity leads to an increase of the error variance, hence of the choice inconsistency. We generate sequential designs, heterogeneous semi-Bayesian designs and homogeneous semi-Bayesian designs considering and ignoring the choice complexity. This way we can examine the advantage of taking the choice complexity into account at the design stage in each design approach.

Simulation results show that the proposed sequential design which takes the choice complexity into account outperforms all other designs we considered. It turns out that the sequential approach generates choice sets with a constant, relatively low complexity level. As the respondents can easily cope with these choice sets, they give consistent choices and these choice sets appear to be most informative about the individual preferences.
\end{abstract}

Keywords: choice complexity, heteroscedastic mixed logit model, individuals' choice consistency, sequential choice designs

\footnotetext{
*This work was supported by the KU Leuven, Belgium.

${ }^{\dagger} \mathrm{KU}$ Leuven, E-mail: Vishva.Danthurebandara@kuleuven. be

${ }^{\ddagger}$ KU Leuven, E-mail: Martina.Vandebroek@kuleuven. be

${ }^{\S}$ KU Leuven, E-mail: Jie.Yu@kuleuven. be
} 


\section{Introduction}

Conjoint choice experiments have become popular for collecting and understanding consumer preferences for product attributes. In such experiments respondents make a sequence of choices. Obtaining informative and consistent choices is crucial to better understand the true preference structure of individuals. The consistency of a decision is affected by several dimensions of the information that is given to the respondents such as information quality, information quantity and the structure of the information (Keller and Staelin (1987)). All these dimensions define the complexity of the choice situation. For example, if the information has high quality, and hence is very useful to the respondent in assessing the utility of an alternative then the choice situation is less complex and the respondent will make consistent choices. On the other hand, if an alternative is described by more attributes than the respondent can cope with, the choice situation is too complex and this leads to inconsistent and inefficient choices. Classical choice models assume that respondents have unlimited information processing capacity which allows them to make their choices in a strictly optimal way irrespective to the complexity of the choice situation (Palma et al. (1994)). A number of studies in psychology and in behavioural science have disproved this assumption and show how the choice complexity negatively affects decision accuracy and effectiveness (for example Keller and Staelin (1987); Wilkie (1974)).

Swait and Adamowicz (2001) consider two rather different ways to model the effect of choice complexity. They argue that not every person has the same capabilities to cope with complexity which leads to variation across individuals. Based on studies in psychology and the behavioural decision theory they argue that the variability across respondents is low when they are given easy choice situations because the best alternative can be chosen without much effort. In medium complex choice sets, the between respondent variability is high since different respondents use different heuristics and effort levels which will make their choices different from each other. When the choice set complexity is high, for instance equal choice probabilities, the variance decreases again compared to the medium complexity scenario because all respondents will choose one of the alternatives with equal utility. They also provide several empirical examples where they model this across respondent variability. Danthurebandara et al. (2011b) followed this idea and showed how the choice complexity affects the efficiency of the conjoint design and the final model estimates. We generated semi-Bayesian D-optimal designs for the heteroscedastic conditional logit model which is parametrized to model the between respondent variability that occurs due to the choice complexity and showed that the designs that are generated while taking the choice complexity into account outperform the designs constructed while ignoring the choice complexity. As far as we know, this is the only study investigating the design efficiency in the context of choice complexity.

On the other hand, Swait and Adamowicz (2001), along with several other authors, describe how within respondent choice inconsistency depends on choice complexity. DeShazo and Fermo (2002) and Sándor and Franses (2009) assume that the decision accuracy monotonically decreases with the 
choice complexity. Therefore an increase in choice complexity leads to a decrease in individual choice consistency. This adverse effect occurs because respondents make mistakes as their cognitive abilities are strained and they revert to simplifying heuristics (DeShazo and Fermo (2002); Palma et al. (1994); Payne et al. (1988)). In their studies in environmental economics and econometrics, DeShazo and Fermo (2002) and Sándor and Franses (2009) provide empirical evidence for this negative effect of the choice complexity on the choice consistency. In this research we follow this idea of modeling the individual inconsistency across choice sets and investigate how the choice complexity affects the efficiency of the conjoint design.

In the papers mentioned above, the heteroscedastic conditional logit model was used which brings the complexity effects into the model through the scale factor. Yet, the heteroscedastic conditional logit model ignores the preference heterogeneity. In this research we extend the heteroscedastic conditional logit model and introduce the heteroscedastic mixed logit model that can cope with both the preference heterogeneity and the scale heterogeneity or the choice consistency. The model in Danthurebandara et al. (2011b) is fundamentally different from the one we use in the current research in two ways. First, the heteroscedastic model we use in the current research is parametrized to model the individuals' choice inconsistency that arises from the choice complexity and not the differences in accuracy across individuals. Second, we use a model that can capture both the preference heterogeneity and the scale heterogeneity. In order to deal with the design problem we propose efficient sequential choice designs (Yu et al. (2011); Danthurebandara et al. (2011a)) generated taking the choice complexity into account. The sequential design approach is one of the most efficient ways to construct choice experiments that are optimized for the mixed logit model. Yu et al. (2011) provide an overview of the difficulties involved in generating optimal designs for mixed logit models. We perform an extensive simulation study where we compare the proposed sequential designs constructed while taking the choice complexity into account with other static designs such as homogeneous semi-Bayesian design (Yu et al. (2008); Danthurebandara et al. (2011b)) and heterogeneous semi-Bayesian design (Sándor and Wedel (2005)) also generated while taking the choice complexity into account. Similar designs constructed while ignoring the choice complexity are considered as benchmarks.

Choosing appropriate measures of choice complexity is another challenge. Several suggestions can be found in the literature. Measures that quantify the information quantity by the number of alternatives and number of attributes are often used. Since these measures are usually fixed in choice experiments, major attention goes to the measures related with the structure of the information such as the number of trade-offs, the average dispersion of attribute values across alternatives and the standard deviation of this alternative specific dispersion. Swait and Adamowicz (2001) argue that all these measures are components of complexity rather than an overall measure and suggest entropy as a direct measure of complexity. We further discuss these measures in section 2.2 where we motivate our choice of complexity measures for the current research.

To sum up, the current research provides methodological contributions. We use a re-parametrized 
mixed logit model which models the individual variation in the error term across choice sets and examine the effect of choice complexity on the efficiency of the design in terms of estimation accuracy. Furthermore, we propose individually adapted sequential choice designs that take the choice complexity into account and study the impact of the choice complexity on the efficiency of the design.

In the next section, we explain the heteroscedastic conditional logit model, the heteroscedastic mixed logit model, the measures of choice complexity and the different design approaches we used. Section 3 presents the simulation study where we compare the different conjoint designs and verify the impact of the choice complexity on their efficiency. Finally, in section 4, we summarise our key findings.

\section{Methodology}

\subsection{Heteroscedastic logit model}

In this paper we consider choice designs for both the heteroscedastic conditional logit model and the heteroscedastic mixed logit model. Therefore, starting from the homoscedastic conditional logit model, we explain the different elements of these models.

Under the assumption of homogeneity in preferences and homoscedasticity, the random utility model is given by

$$
U_{k s n}=\mathbf{x}_{k s n}^{\prime} \boldsymbol{\beta}+\varepsilon_{k s n},
$$

where the $p$-dimensional vector $\mathbf{x}_{k s n}$ contains the attribute values of alternative $k$ from choice set $s$ for respondent $n, \boldsymbol{\beta}$ is a $p$-dimensional vector of utility coefficients and $\varepsilon_{k s n}$ is a Gumbel distributed error term. For a given respondent $n$, the probability of choosing alternative $k$ from choice set $s$ is given by

$$
p_{k s n}^{c l}(\boldsymbol{\beta})=\frac{\exp \left(\mu \mathbf{x}_{k s n}^{\prime} \boldsymbol{\beta}\right)}{\sum_{i=1}^{K} \exp \left(\mu \mathbf{x}_{i s n}^{\prime} \boldsymbol{\beta}\right)} .
$$

The parameter $\mu$ is called the scale parameter because it scales the utility coefficients to reflect the error variance. The scale parameter and the utility coefficients are not separately identified (Train (2003)). The scale can be chosen to provide a convenient value for the error variance. Under the

Gumbel distributed errors, the scale parameter is equal to $\frac{\pi}{\sqrt{6} \sigma}$, where $\sigma$ is the error variance. The model which assumes constant error variance is known as the homoscedastic conditional logit model or simply the conditional logit model.

The error variance $\sigma$ quantifies the degree of the individual's choice inconsistency. For example, high choice consistency is reflected by a small error variance. Hence, the error variance measures the choice inconsistency, and the scale parameter, which is inversely proportional to the error variance, is a direct 
indicator of the choice consistency. We model this scale heterogeneity as $\mu_{s}(\boldsymbol{\theta})=e^{\mathbf{c}_{s}^{\prime} \boldsymbol{\theta}}$, where $\mathbf{c}_{s}$ is the vector of complexity measurements of choice set $s$ and $\boldsymbol{\theta}$ is the $q$-dimensional vector of complexity coefficients. Then the choice probability is given by

$$
p_{k s n}^{h c l}(\boldsymbol{\beta}, \boldsymbol{\theta})=\frac{\exp \left(\mu_{s}(\boldsymbol{\theta}) \mathbf{x}_{k s n}^{\prime} \boldsymbol{\beta}\right)}{\sum_{i=1}^{K} \exp \left(\mu_{s}(\boldsymbol{\theta}) \mathbf{x}_{i s n}^{\prime} \boldsymbol{\beta}\right)} .
$$

This is called the heteroscedastic conditional logit model. The likelihood for the heteroscedastic conditional logit model model can be written as

$$
L^{h c l}(\mathbf{Y} \mid \mathbf{X}, \boldsymbol{\beta}, \boldsymbol{\theta})=\prod_{n=1}^{N} \prod_{s=1}^{S} \prod_{k=1}^{K}\left(p_{k s n}^{h c l}(\boldsymbol{\beta}, \boldsymbol{\theta})\right)^{y_{k s n}}
$$

where $y_{k s n}$ is a binary indicator that equals one if respondent $n$ chooses alternative $k$ from choice set $s$ and zero otherwise, and $\mathbf{Y}=\left[\mathbf{y}_{1}, \ldots, \mathbf{y}_{N}\right]$ is the matrix of choices from $N$ respondents. This heteroscedastic conditional logit model is used in several other studies in this context, for instance, Swait and Adamowicz (2001), DeShazo and Fermo (2002), Sándor and Franses (2009) and Danthurebandara et al. (2011b).

The heteroscedastic conditional logit model assumes preference homogeneity, that is, all respondents share the same utility coefficients $\boldsymbol{\beta}$. In contrast, the mixed logit model, which assumes individual specific taste coefficients, is often used to model the market heterogeneity (for example Bliemer and Rose (2010); Yu et al. (2011)). In this research we extend the heteroscedastic conditional logit model to incorporate the preference heterogeneity. Moreover, respondents cope with complex situations based on their own cognitive abilities and hence, the effect of choice complexity is different from individual to individual. Therefore, it is quite logical also to assume heterogeneity in the complexity coefficients.

Let $\boldsymbol{\beta}_{n}$ and $\boldsymbol{\theta}_{n}$ be the $p$-dimensional utility coefficient vector and the $q$-dimensional complexity coefficient vector specific to respondent $n$, respectively. Then, conditional on $\boldsymbol{\beta}_{n}$ and on $\boldsymbol{\theta}_{n}$, the probability that respondent $n$ chooses alternative $k$ from choice set $s$ is given by

$$
p_{k s n}^{h m l}\left(\boldsymbol{\beta}_{n}, \boldsymbol{\theta}_{n}\right)=\frac{\exp \left(\mu_{s n}\left(\boldsymbol{\theta}_{n}\right) \mathbf{x}_{k s n}^{\prime} \boldsymbol{\beta}_{n}\right)}{\sum_{i=1}^{K} \exp \left(\mu_{s n}\left(\boldsymbol{\theta}_{n}\right) \mathbf{x}_{i s n}^{\prime} \boldsymbol{\beta}_{n}\right)},
$$

where $\mu_{s n}\left(\boldsymbol{\theta}_{n}\right)=e^{\mathbf{c}_{s}^{\prime} \boldsymbol{\theta}_{n}}$. For a given respondent $n$, the individual likelihood is

$$
L^{h m l}\left(\mathbf{y}_{n} \mid \mathbf{x}_{n}, \boldsymbol{\beta}_{n}, \boldsymbol{\theta}_{n}\right)=\prod_{s=1}^{S} \prod_{k=1}^{K}\left(p_{k s n}^{h m l}\left(\boldsymbol{\beta}_{n}, \boldsymbol{\theta}_{n}\right)\right)^{y_{k s n}}
$$

where $\mathbf{x}_{n}$ is the full choice design that respondent $n$ evaluated and $\mathbf{y}_{n}$ contains the series of choices that respondent $n$ made. In order to capture the respondents' preference heterogeneity and the scale heterogeneity, the individual specific utility coefficient vector $\boldsymbol{\beta}_{n}$ and the individual specific complexity coefficient vector $\boldsymbol{\theta}_{n}$ are assumed to be normally distributed, that is $\boldsymbol{\beta}_{n} \sim \phi\left(\boldsymbol{\beta}_{n} \mid \boldsymbol{\mu}_{\beta}, \boldsymbol{\Sigma}_{\beta}\right)$ and $\boldsymbol{\theta}_{n} \sim$ 
$\phi\left(\boldsymbol{\theta}_{n} \mid \boldsymbol{\mu}_{\theta}, \boldsymbol{\Sigma}_{\theta}\right)$. Then the individual likelihood, unconditional on $\boldsymbol{\beta}_{n}$ and $\boldsymbol{\theta}_{n}$, is given by

$$
\begin{array}{r}
L^{h m l}\left(\mathbf{y}_{n} \mid \mathbf{x}_{n}, \boldsymbol{\mu}_{\beta}, \boldsymbol{\Sigma}_{\beta}, \boldsymbol{\mu}_{\theta}, \boldsymbol{\Sigma}_{\theta}\right)=\iint L^{h m l}\left(\mathbf{y}_{n} \mid \mathbf{x}_{n}, \boldsymbol{\beta}_{n}, \boldsymbol{\theta}_{n}\right) \phi\left(\boldsymbol{\beta}_{n} \mid \boldsymbol{\mu}_{\beta}, \boldsymbol{\Sigma}_{\beta}\right) \phi\left(\boldsymbol{\theta}_{n} \mid \boldsymbol{\mu}_{\theta}, \boldsymbol{\Sigma}_{\theta}\right) d \boldsymbol{\theta}_{n} d \boldsymbol{\beta}_{n} \\
=\iint\left(\prod_{s=1}^{S} \prod_{k=1}^{K}\left(p_{k s n}^{h m l}\left(\boldsymbol{\beta}_{n}, \boldsymbol{\theta}_{n}\right)\right)^{y_{k s n}}\right) \phi\left(\boldsymbol{\beta}_{n} \mid \boldsymbol{\mu}_{\beta}, \boldsymbol{\Sigma}_{\beta}\right) \phi\left(\boldsymbol{\theta}_{n} \mid \boldsymbol{\mu}_{\theta}, \boldsymbol{\Sigma}_{\theta}\right) d \boldsymbol{\theta}_{n} d \boldsymbol{\beta}_{n}
\end{array}
$$

This model is called the heteroscedastic mixed logit model and the corresponding likelihood of the observed choices of $N$ respondents is

$$
L^{h m l}\left(\mathbf{Y} \mid \mathbf{X}, \boldsymbol{\mu}_{\beta}, \boldsymbol{\Sigma}_{\beta}, \boldsymbol{\mu}_{\theta}, \boldsymbol{\Sigma}_{\theta}\right)=\prod_{n=1}^{N} L^{h m l}\left(\mathbf{y}_{n} \mid \mathbf{x}_{n}, \boldsymbol{\mu}_{\beta}, \boldsymbol{\Sigma}_{\beta}, \boldsymbol{\mu}_{\theta}, \boldsymbol{\Sigma}_{\theta}\right)
$$

where $\mathbf{Y}$ contains the responses for all $N$ respondents and $\mathbf{X}$ is the full design which is the collection of $N$ sub-designs that are evaluated by the $N$ respondents.

\subsection{Measures of choice complexity}

Several measures to quantify the choice complexity can be found in the literature. Swait and Adamowicz (2001) introduce entropy as a single measure of complexity. Entropy measures the uncertainty of a choice situation and is therefore a direct measure of complexity, meaning that high entropy indicates high complexity. Entropy for a given choice set s can be defined as

$$
H_{s}=-\sum_{k=1}^{K} p_{k s n}^{c l}(\boldsymbol{\beta}) \ln p_{k s n}^{c l}(\boldsymbol{\beta})
$$

with $p_{k s n}^{c l}(\boldsymbol{\beta})$ as defined in (2.2). By definition, entropy depends on the utility coefficients.

Other authors use components that measure the quantity of information and the structure of information. Table 1 gives a chronological list of some papers, together with the complexity measures involved.

The information quantity is often measured by the number of alternatives per choice set and the number of attributes per choice set. However, most of the time these two measures are designed to be fixed in choice experiments. Therefore, measures of the structure of information are widely used to quantify the choice complexity in a design context. We discuss the three main measures.

- Number of trade-offs: this measures the similarity of alternatives in terms of utility and was introduced by Sándor and Franses (2009). Let $\boldsymbol{d}$ be a vector of signs of the difference of the pair of alternatives we wish to compare: $\boldsymbol{d}=\left(\operatorname{sign}\left(x_{11}-x_{21}\right), \ldots, \operatorname{sign}\left(x_{1 p}-x_{2 p}\right)\right)$ where $x_{k p}$ is the value of the attribute $p$ in alternative $k$. $\operatorname{Sign}\left(x_{11}-x_{21}\right)$ equals 1 if $\left(x_{11}-x_{21}\right)>0$, equals 0 if $\left(x_{11}-x_{21}\right)=0$ and equals -1 if $\left(x_{11}-x_{21}\right)<0$. If the number of negative signs in $\boldsymbol{d}$ is 
Table 1: Overview of some papers together with the complexity measures and the designs involved

\begin{tabular}{|c|c|c|}
\hline Author & Complexity measures & Design \\
\hline Mazzotta and Opaluch (1995) & $\begin{array}{l}\text {-Number of attributes that } \\
\text { vary across the alternatives }\end{array}$ & Factorial design \\
\hline Dellaert et al. (1999) & $\begin{array}{l}\text {-Attribute level difference } \\
\text {-Absolute attribute levels }\end{array}$ & Factorial design \\
\hline DeShazo and Fermo (2002) & $\begin{array}{l}\text {-Number of alternatives } \\
\text {-Number of attributes } \\
\text {-Number of attributes that } \\
\text { vary across the alternatives } \\
\text {-Mean standard deviation of } \\
\text { attributes for each alternative } \\
\text { in the choice set } \\
\text {-Dispersion of the standard } \\
\text { deviation of each alternative } \\
\text { in the choice set }\end{array}$ & $\begin{array}{l}\text { Designs generated with } \\
\text { random variation in five } \\
\text { complexity measures }\end{array}$ \\
\hline Sándor and Franses (2009) & $\begin{array}{l}\text {-Mean standard deviation of } \\
\text { attributes for each alternative } \\
\text { in the choice set } \\
\text {-Number of trade-offs } \\
\text {-Price discount (dummy) }\end{array}$ & $\begin{array}{l}\text { Heterogeneous semi-Bayesian } \\
\text { design }\end{array}$ \\
\hline Dellaert et al. (2012) & $\begin{array}{l}\text {-Number of alternatives } \\
\text {-Number of attributes } \\
\text {-Utility similarity }\end{array}$ & Factorial designs \\
\hline
\end{tabular}

larger than the number of positive signs then $\boldsymbol{d}$ is replaced by $-\boldsymbol{d}$. The number of trade-offs is defined as $(a-b)$ where $a$ is the number of non-zero components of $\boldsymbol{d}$ and $b$ is the number of positive components. This measure is expected to have a negative effect on the scale parameter since an increase in the number of trade-offs leads to an increase in complexity that should be reflected by a higher error variance. A measure similar to this one is used by DeShazo and Fermo (2002) and was called the number of attributes whose levels differ across alternatives, but Sándor and Franses (2009) argue that their measure of trade-offs captures the degree of complexity in a choice situation better, especially in the presence of dominant alternatives.

- Average $S D$ : this measures the average variability of alternatives and is defined as

$$
\text { Average } S D=\frac{S D_{1}+\ldots+S D_{K}}{K}
$$

where $S D_{k}=\sqrt{\frac{1}{p} \sum_{i=1}^{p}\left(x_{k i}-\bar{x}_{k}\right)^{2}}$ is the standard deviation of the attribute levels of alternative $k, \bar{x}_{k}$ is the mean attribute value of alternative $k$ and $K$ and $p$ are the total number of alternatives in the choice set and the total number of attributes. This measure is also expected to have a negative impact on the scale parameter. If all the attribute levels take high values then the variability is low and $S D_{k}$ takes a low value. Alternatively, if some attribute levels take high 
values and others take low values then the respondent has to make many trade-offs and $S D_{k}$ will be high. Therefore, high values of the Average SD indicate high complexity and hence have a negative impact on the scale.

- Dispersion $S D$ : this is the standard deviation of $S D_{k}$ across alternatives and is defined as

$$
\text { Dispersion } S D=\sqrt{\frac{1}{K} \sum_{k=1}^{K}\left(S D_{k}-\text { Average } S D\right)^{2}} \text {. }
$$

A high value of this measure indicates high dispersion across alternatives and is expected to have a negative effect on the scale.

Unlike entropy, these three complexity measures depend only on the design characteristics and not on the utility coefficients. To compare these two types of complexity measures (entropy and the measures of the structure of information) we used an empirical example presented in Bierlaire and Ben-Akiva (2010) where they discuss the Swiss-Metro project. The Swiss-Metro project is a choice experiment which was conducted in Switzerland in March 1998 to evaluate the respondent preferences for different travel modes. We use preference data from 200 respondents who were assigned to random designs which each consist of nine choice sets with three alternatives. We estimate the heteroscedastic mixed logit model using the entropy and the other three complexity measures. For the first model, the scale parameter is given by $\mu_{s n}\left(\boldsymbol{\theta}_{n}\right)=\exp \left(\theta_{n}\right.$ entropy $\left._{s}\right)$ and for the second model it is $\mu_{s n}\left(\boldsymbol{\theta}_{n}\right)=$ $\exp \left(\theta_{n 1}\right.$ trade-offs $s_{s}+\theta_{n 2}$ average $\mathrm{SD}_{s}+\theta_{n 3}$ dispersion $\left.\mathrm{SD}_{s}\right)$. Table 2 shows the deviance information criterion (DIC) values and the prediction accuracies of the two models. The DIC value is a compromise between the model fit as measured by the expected deviance and the complexity of the model as measured by the effective number of parameters. To assess the prediction accuracy, we computed how often the choice of the individual respondents for the holdout choice set is predicted correctly by each model (hit rate). The results show that the model with the number of trade-offs, the average SD and

Table 2: Comparison of complexity measures

\begin{tabular}{ccc}
\hline Model & DIC & Hit rate \\
\hline Model with entropy & 2164.69 & $80 \%$ \\
Model with complexity measures & 2074.46 & $85 \%$ \\
\hline
\end{tabular}

the dispersion SD performs better with respect to both model fit and prediction accuracy, than the model based on entropy. Though we can not generalize this result based on one case study, the model with the number of trade-offs, the average SD and the dispersion SD seems realistic and we use these to measure the choice complexity in this research. 


\subsection{Design construction methods}

The third column in Table 1 shows the design that was used in the corresponding paper to collect the data. It shows that only one paper (DeShazo and Fermo (2002)) incorporates the effect of choice complexity during the design stage. In their empirical study in environmental economics, DeShazo and Fermo (2002) generated choice sets with random variation in their five complexity measures. Factorial designs and orthogonal designs that are not optimized for a certain model have often been used. Sándor and Franses (2009) use a heterogeneous semi-Bayesian design, which we also use in the current research as a benchmark design, but they constructed this design ignoring the choice complexity. In this research we propose an individually adapted sequential design approach that generates the next choice set for a given respondent based on his/her previous responses. The sequential approach is compared with standard heterogeneous semi-Bayesian design and homogeneous semi-Bayesian design approaches. We construct these designs for the models that take the choice complexity into account as well as for the models that ignore the complexity and compare the design efficiency with respect to the estimation accuracy. The next sections present a brief explanation of these design construction methods.

\subsubsection{Individual sequential design approach}

We propose sequentially optimized individual designs for the heteroscedastic mixed logit model. The use of sequential designs for mixed logit models is motivated by the findings of Yu et al. (2011). The (heteroscedastic) mixed logit model assumes that every individual respondent follows a (heteroscedastic) conditional logit model and combines these individual logit models into a population-level model. The sequential design approach allows to construct optimal designs for the heteroscedastic conditional logit model for each respondent. The general idea of this approach is to construct the next choice set for a given respondent in a Bayesian way based on the previous responses of that particular respondent. As such, each respondent gets choice sets which are fully adapted to the individual's preference and complexity structure as known at that moment.

The sequential design approach consists of an initial static stage and a sequential stage. In the initial stage each respondent has to evaluate a few initial choice sets that are generated using a common prior distribution. The methodological paper of Yu et al. (2011) which introduced the sequential design approach to the choice experiment literature shows that using an initial static stage with few choice sets allows more reliable results than starting the sequential approach from the beginning. These initial choice data are analysed in a Bayesian way and yield a posterior distribution on the preference and complexity parameters. This posterior is used as the prior distribution to generate the next choice set. Once the respondent has evaluated the new choice set, the prior is updated with all choices until the current stage and the new posterior distribution is obtained. The updated posterior distribution is used to generate the next choice set. Appendix A further explains the design criterion and the design generation algorithm in more mathematical detail. 
In order to assess the effect of choice complexity on the design efficiency, we also generate sequential designs for the homoscedastic mixed logit model. In this way we can compare the performance of designs that are constructed for models with and without choice complexity effects. More details about constructing sequential designs based on the homoscedastic mixed logit model can be found in Yu et al. (2011).

\subsubsection{Semi-Bayesian D-optimal designs}

Semi-Bayesian designs are constructed based on the heteroscedastic conditional logit model using a common and fixed prior for the utility coefficients and for the complexity coefficients. In our simulation study this common prior is the one we use as the initial prior in the sequential design approach. These designs are generated in a non-Bayesian maximum likelihood context and the design prior is only taken into account at the design stage, hence it is called a semi-Bayesian design criterion. In this research we use two types of semi-Bayesian designs: a homogeneous semi-Bayesian design and a heterogeneous semi-Bayesian design.

The first design approach ignores the market heterogeneity completely and every respondent has to evaluate the same design (Yu et al. (2008)). For more details about constructing semi-Bayesian designs for the heteroscedastic conditional logit model we refer to Danthurebandara et al. (2011b).

The heterogeneous semi-Bayesian approach was introduced by Sándor and Wedel (2005) to estimate the homoscedastic mixed logit model. Here different designs are used for each respondent. Generating heterogeneous designs is computationally time consuming since $N$ different designs have to be generated by optimizing certain design criterion sequentially, for example the $\mathrm{D}$ design criterion in the current study (refer to Kessels et al. (2006) and Appendix A for more details about the D criterion). Sándor and Wedel (2005) show that $M=6$ different designs give approximately the same efficiency as $N$ different designs. The greedy approach is used to determine $M$ designs sequentially as follows:

$$
\begin{gathered}
\min _{X_{1}} D(X) \text { to obtain } X_{1} \\
\min _{X_{2}} D\left(X_{1}, X\right) \text { to obtain } X_{2} \\
\vdots \\
\min _{X_{M}} D\left(X_{1}, \ldots, X\right) \text { to obtain } X_{M}
\end{gathered}
$$

We generate 20 different semi-Bayesian designs sequentially using the greedy approach and randomly assign them to the $N$ respondents. For further details about generating heterogeneous design using the greedy approach we refer to Sándor and Wedel (2005). Similar to the sequential approach, the semi-Bayesian designs that are constructed without complexity effects in the model are also considered as benchmark designs. 


\subsection{Estimation}

After collecting data using the sequential approach, the homogeneous semi-Bayesian designs and the heterogeneous semi-Bayesian designs that are optimized for the models with and without complexity effects, we estimate the heteroscedastic mixed logit model using the hierarchical Bayes approach. The Bayesian procedure is convenient to use and has often been used in the recent discrete choice literature. One of the major advantages of this approach is that it can provide both individual-level parameter estimates and population-level parameter estimates. Furthermore, Bayesian methods allow us to avoid complex optimization problems that can occur in classical maximum likelihood methods. Train (2003) gives a detailed theoretical explanation about the hierarchical Bayes method for estimating the mixed logit models and several implementations of this method can be found in the literature, for example in Yu et al. (2011) and in Danthurebandara et al. (2011a).

\section{Simulation study}

We evaluate the efficiency of the different designs to estimate the heteroscedastic mixed logit model in a simulation study. Table 3 lists the designs we compared. Profiles are characterized by two attributes

Table 3: Different designs considered in the simulation study

\begin{tabular}{lll}
\hline & Design & Choice model \\
\hline$S e q^{c}$ & Sequential & Heteroscedastic mixed logit model \\
$S e q$ & Sequential & Homoscedastic mixed logit model \\
$S B_{\text {hetro }}^{c}$ & Heterogenous semi-Bayesian & Heteroscedastic conditional logit model \\
$S B_{\text {hetro }}$ & Heterogenous semi-Bayesian & Homoscedastic conditional logit model \\
$S B_{\text {homo }}^{c}$ & Homogenous semi-Bayesian & Heteroscedastic conditional logit model \\
$S B_{\text {homo }}$ & Homogenous semi-Bayesian & Homoscedastic conditional logit model \\
\hline
\end{tabular}

with three levels and one attribute with two levels. Designs include 12 choice sets and three alternatives per choice set $\left(3^{2} \times 2^{1} / 3 / 12\right)$. We apply effect coding (Yu et al. (2008)) to the attributes resulting in five distinct utility coefficients and we assume that the individual parameter vectors $\boldsymbol{\beta}_{n}=\left[\beta_{1 n}, \beta_{2 n}\right.$, $\left.\beta_{3 n}, \beta_{4 n}, \beta_{5 n}\right]$ and $\boldsymbol{\theta}_{n}=\left[\theta_{1 n}, \theta_{2 n}, \theta_{3 n}\right]$ are independent and follow multivariate normal distributions, that is $\boldsymbol{\beta}_{n} \sim N\left(a_{\beta} \overline{\boldsymbol{\beta}}, \sigma_{\beta} \boldsymbol{\Sigma}_{\beta}\right)$ and $\boldsymbol{\theta}_{n} \sim N\left(a_{\theta} \overline{\boldsymbol{\theta}}, \sigma_{\theta} \boldsymbol{\Sigma}_{\theta}\right)$ where $\overline{\boldsymbol{\beta}}=[-1,0,-1,-1,0], \boldsymbol{\Sigma}_{\beta}=\mathbf{I}_{5}, \overline{\boldsymbol{\theta}}=$ $[-0.2,-1.4,-1]$ and $\boldsymbol{\Sigma}_{\theta}=\operatorname{diag}(0.1,0.5,0.3)$. The mean values for the complexity parameters are taken from an empirical application presented in DeShazo and Fermo (2002). We vary the mean and the heterogeneity of both the utility coefficients and the complexity coefficients by setting them at low and high levels. Table 4 shows the 16 combinations (scenarios) we considered in the simulation study.

In each scenario 200 respondents are considered. To generate sequential designs, 5 choice sets are used in the initial stage and 7 choice sets are generated sequentially. In each case, $\boldsymbol{\beta}_{n} \sim N\left(a_{\beta} \overline{\boldsymbol{\beta}}, \sigma_{\beta} \boldsymbol{\Sigma}_{\beta}\right)$ 
Table 4: Different scenarios considered in the simulation study

\begin{tabular}{|c|c|c|c|c|c|c|}
\hline & & & \multicolumn{4}{|c|}{$\boldsymbol{\theta}_{n}$} \\
\hline & & & \multicolumn{2}{|c|}{$a_{\theta}=0.5$ (low) } & \multicolumn{2}{|c|}{$a_{\theta}=1$ (high) } \\
\hline & & & $\begin{array}{l}\sigma_{\theta}=0.25 \\
\text { (low) }\end{array}$ & $\begin{array}{l}\sigma_{\theta}=1 \\
(\text { high })\end{array}$ & $\begin{array}{l}\sigma_{\theta}=0.5 \\
\text { (low) }\end{array}$ & $\begin{array}{l}\sigma_{\theta}=2 \\
\text { (high) }\end{array}$ \\
\hline \multirow{4}{*}{$\boldsymbol{\beta}_{n}$} & \multirow{2}{*}{$a_{\beta}=0.5$ (low) } & $\sigma_{\beta}=0.25$ (low) & 1 & 2 & 3 & 4 \\
\hline & & $\sigma_{\beta}=1$ (high) & 5 & 6 & 7 & 8 \\
\hline & \multirow{2}{*}{$a_{\beta}=1$ (high) } & $\sigma_{\beta}=0.5($ low $)$ & 9 & 10 & 11 & 12 \\
\hline & & $\sigma_{\beta}=2$ (high) & 13 & 14 & 15 & 16 \\
\hline
\end{tabular}

and $\boldsymbol{\theta}_{n} \sim N\left(a_{\theta} \overline{\boldsymbol{\theta}}, \sigma_{\theta} \boldsymbol{\Sigma}_{\theta}\right)$ are used as the initial prior distributions of the model parameters. The same prior distribution is used to generate the semi-Bayesian designs. We use this prior distribution also to generate the individual choices. This assumes that the prior distribution that is used to generate the designs contains perfect information about the true heterogeneity distribution of the model parameters. This assumption is of course not realistic. Appendix $\mathrm{C}$ presents a robustness study where we conduct a simulation study by relaxing this assumption and assess the design performance in different levels of prior misspecification. The main results that are presented here for the perfect information are also valid under prior misspecification. All design construction algorithms and hierarchical Bayes estimation are implemented in SAS/IML version 9.3.

To visualize how well each design approach can estimate the true heterogeneity distribution of the utility coefficients and the complexity coefficients, we constructed beanplots which allow us to compare the estimated heterogeneity distributions with the true heterogeneity distribution (Kampstra (2008)). In Figure 1 and Figure 2, each bean consist of two densities: on the left hand side the true heterogeneity density is shown and on the right hand side the heterogeneity density estimated by a particular design approach is given. These density traces are combined with a 1-dimensional scatter plot of individual observations which are represented by short horizontal white lines. Figure 1 corresponds to the case of low mean and low heterogeneity in both the utility coefficients and the complexity coefficients. Figure 2 corresponds to the case of low mean and low heterogeneity in the utility coefficients and high mean and high heterogeneity in the complexity coefficients.

These figures show that the sequential designs allow much better estimation of the true heterogeneity distribution of both the utility coefficients and the complexity coefficients than the other non-sequential designs. Among the sequential designs, the design that is constructed for the heteroscedastic mixed logit model has the best performance, but even for the homoscedastic mixed logit model, the sequential approach is significantly outperforming the other approaches. Moreover, designs that take the complexity into account allow better estimation of the heterogeneity distribution of the complexity coefficients than the designs that ignore the complexity. The homogeneous semi-Bayesian designs fail to capture the true preference heterogeneity and the scale heterogeneity completely regardless whether the complexity was taken into account or not. The difference between the designs that are constructed 


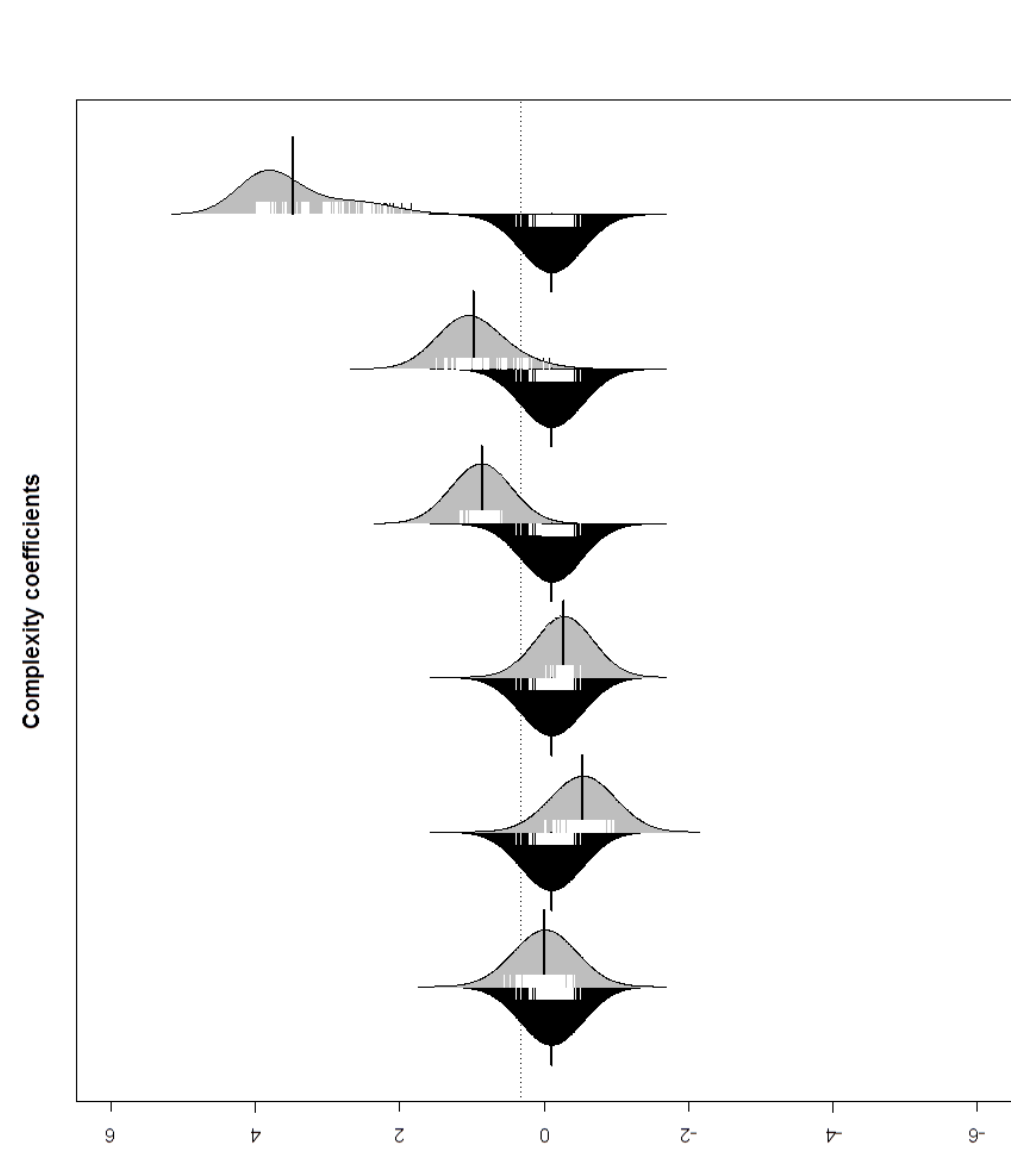

$\exists \stackrel{\Xi}{\square}$

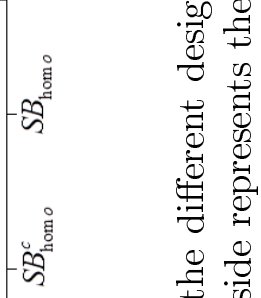

。 青营

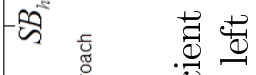

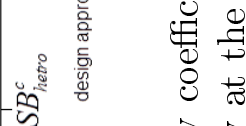

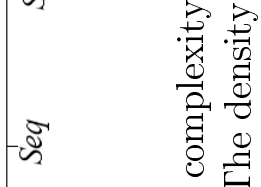

F

\%

爱峞

.8.

$\notin B$

这

금

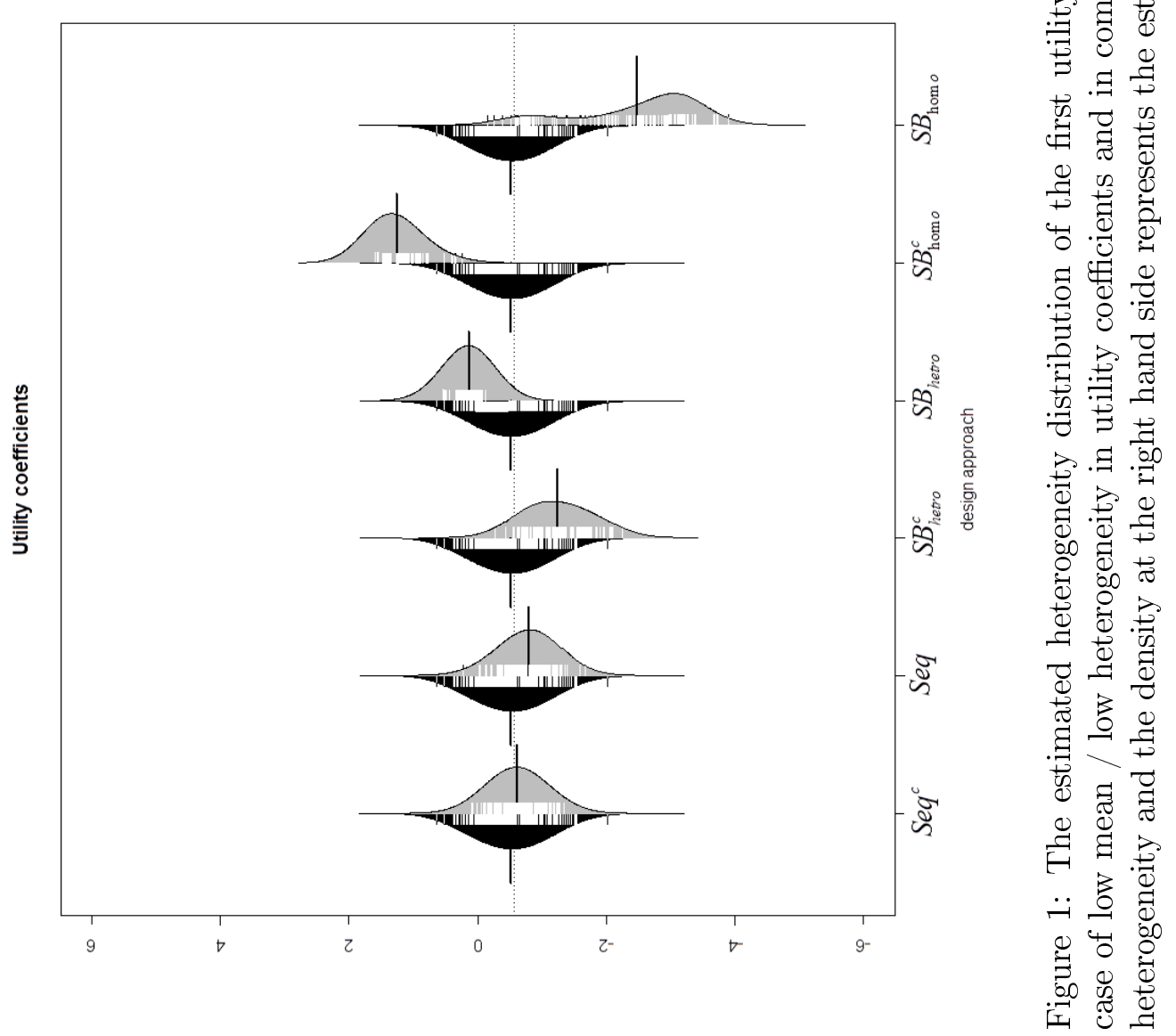




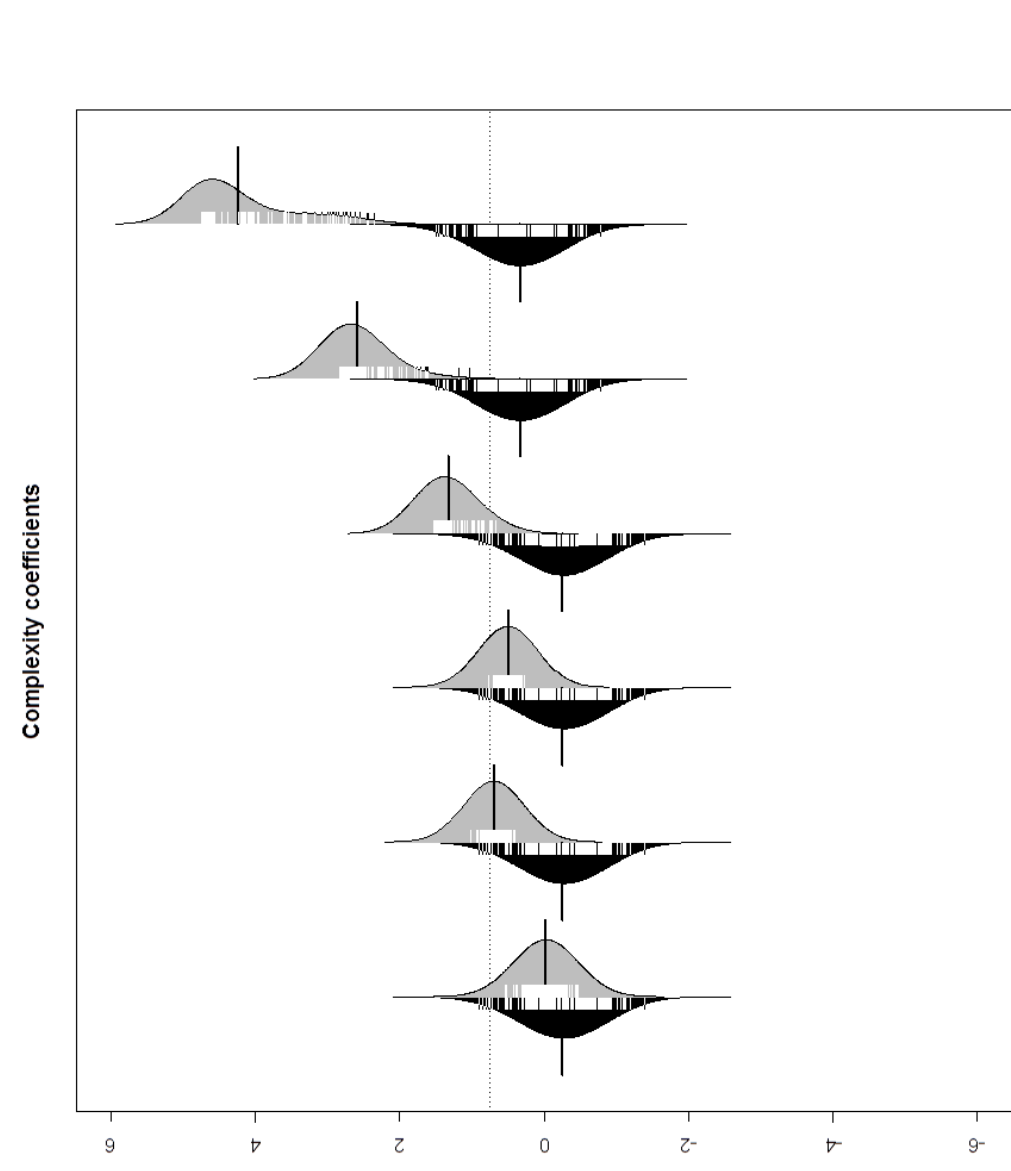

莎

.$\Xi \underset{\Xi}{ \pm}$

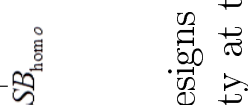

की $\quad \overrightarrow{0}$

임

药 离

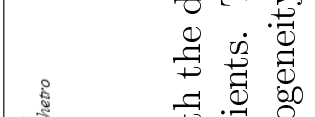

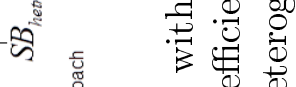

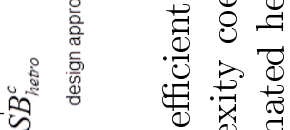

की

究

\%

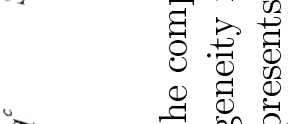

\%

范

政

过

记

0 는

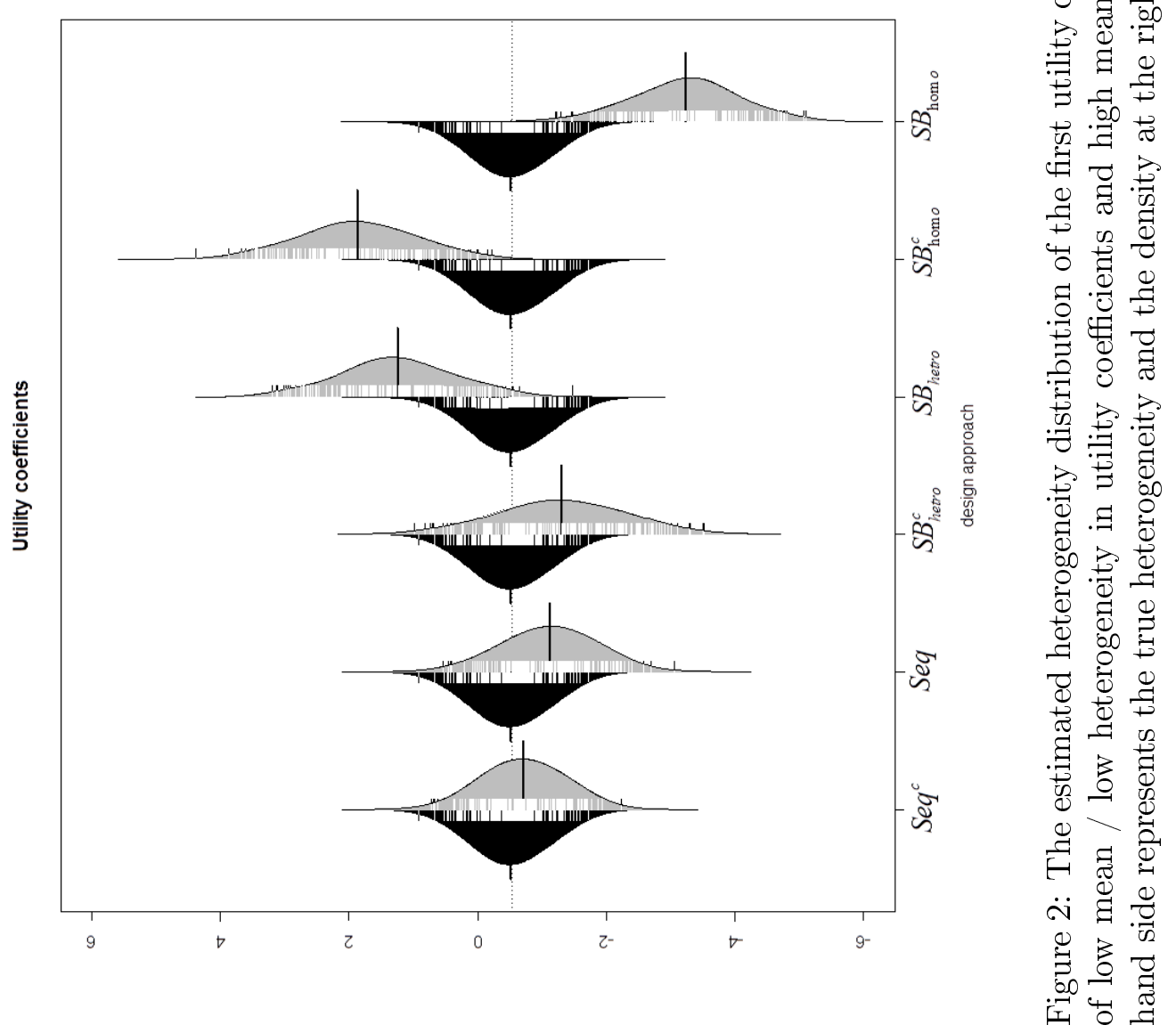


considering the complexity and the designs constructed ignoring the complexity is much higher in the case of low mean and low heterogeneity in complexity coefficient compared to the case of high mean and high heterogeneity in complexity coefficient. This plot also suggests that the sequential optimization of the designs is at least as important as taking the complexity into account.

We evaluate the performance of the designs presented in Table 3 also based on the accuracy of the population parameter estimates. This is assessed by the root mean squared error (RMSE) which quantifies how close the parameter estimates are to the true parameters (Arora and Huber (2001); Toubia et al. (2004)). For each design, the estimation accuracy of the population mean $\left(\boldsymbol{\mu}_{\beta}\right)$, the population covariance $\left(\boldsymbol{\Sigma}_{\beta}\right)$ and the individual-level utility coefficients $\left(\boldsymbol{\beta}_{n}\right)$ are assessed. The RMSE values obtained under different design approaches and for the 16 scenarios we considered in the simulation study are tabulated in Appendix B. In order to visualize how well the population-level parameters are estimated under the different design approaches, we plot the estimated population mean and the population variance of the utility coefficients (Figure 3) and the complexity coefficients (Figure 4) for the case of low response accuracy and low heterogeneity in both the utility and complexity effects. In Figure 3 and Figure 4, black points connected by a solid line represent the true parameter values.

These figures show that the sequential design constructed considering the complexity effects allow more accurate estimation of the population-level preference parameters and complexity parameters more accurately compared to the other designs.

We now examine how complex the choice sets are that are generated by the different design approaches. We calculate the scale parameter for each choice set and for each respondent by the following expression:

$$
\boldsymbol{\mu}_{s n}\left(\boldsymbol{\theta}_{n}\right)=\exp \left(\theta_{1 n} \times \text { trade-offs }_{s}+\theta_{2 n} \times \text { average } \mathrm{SD}_{s}+\theta_{3 n} \times \text { dispersion } \mathrm{SD}_{s}\right),
$$

where $\boldsymbol{\theta}_{n}=\left(\theta_{1 n}, \theta_{2 n}, \theta_{3 n}\right)$ is the vector of true complexity coefficients. Then the error variance, which is inversely proportional to the scale parameter, is calculated for each choice set and for each respondent. Figure 5 shows the error variances averaged over all respondents for each choice set generated by the different design approaches.

The designs that are constructed taking the choice complexity into account have smaller error variance than the designs generated by the similar approach while ignoring the choice complexity and hence the former designs yield more consistent choices. This is very clear for the sequential approach and for the heterogeneous semi-Bayesian designs. Remark that the sequential approach generates choice sets that have approximately the same error variance. Panel (a) in Figure 5 shows that the sequential approach starts generating choice sets with approximately the same error variance immediately after starting the adaptive sequential stage (choice set 6 to choice set 12). In order to examine the distribution of the true error variance over all the possible choice sets, we generated all 816 possible choice sets under the design setting $3^{2} \times 2^{1} / 3 / 12$. For each respondent and for each choice set, the error variance was 


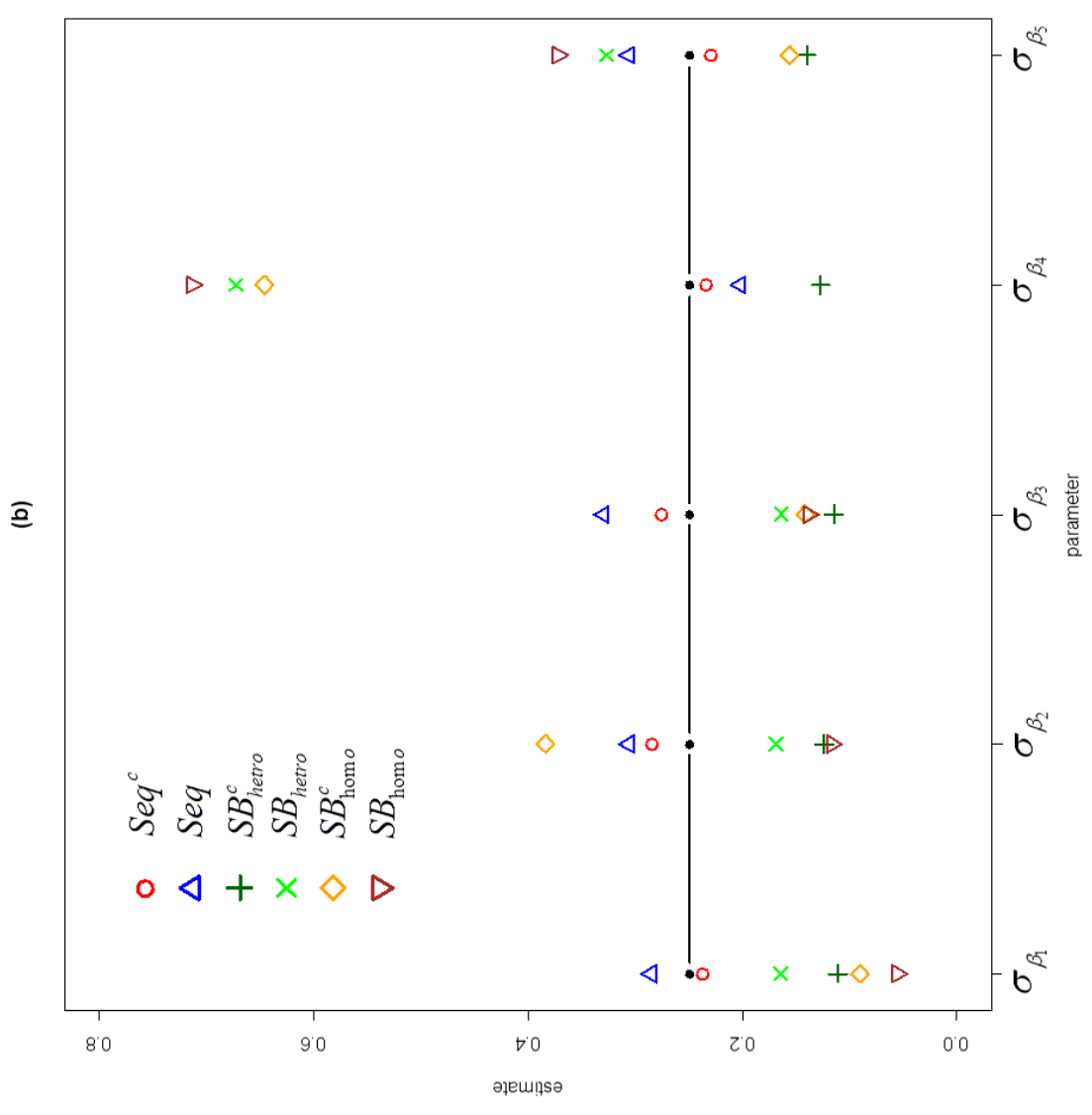

ल

ป

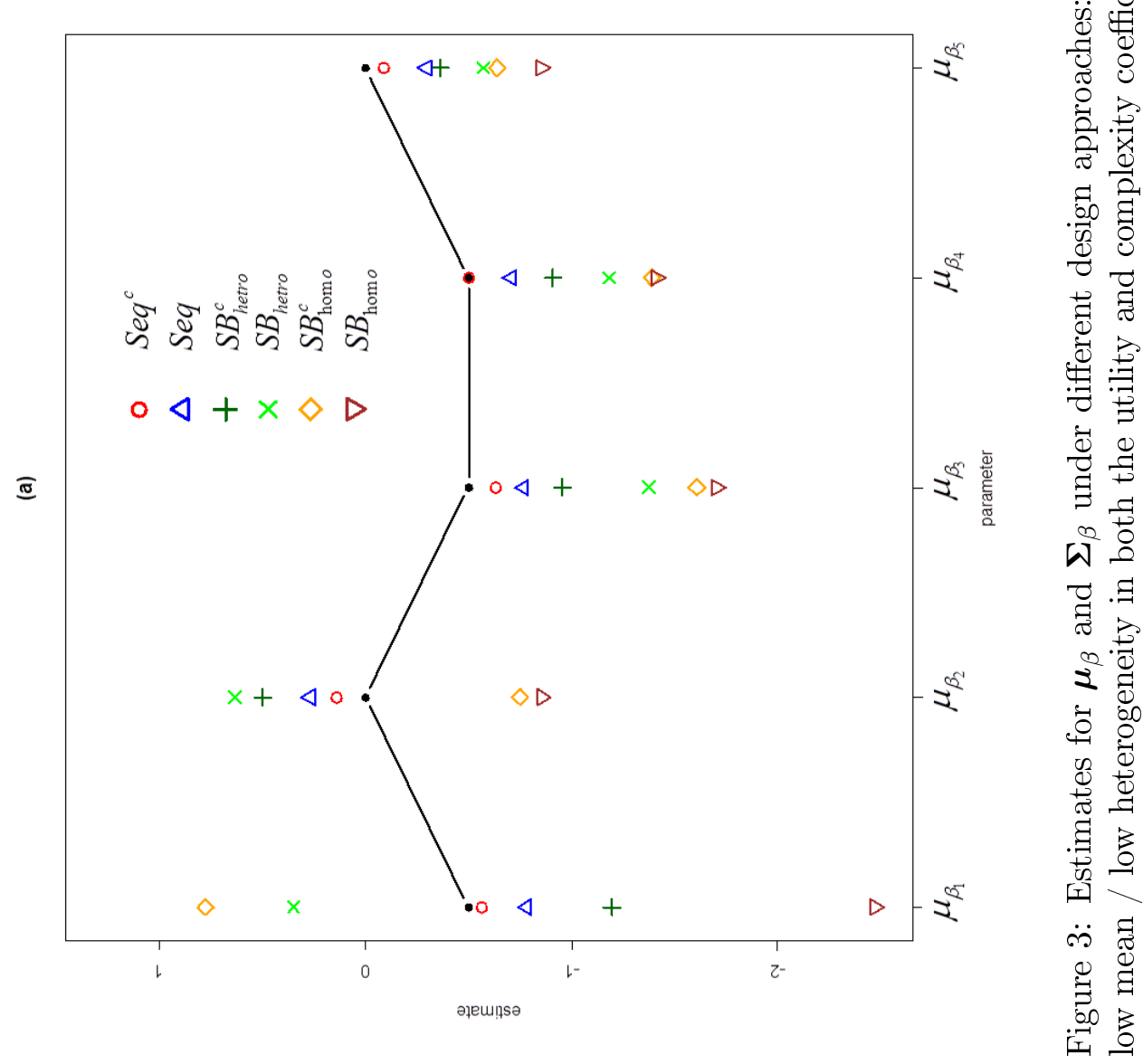




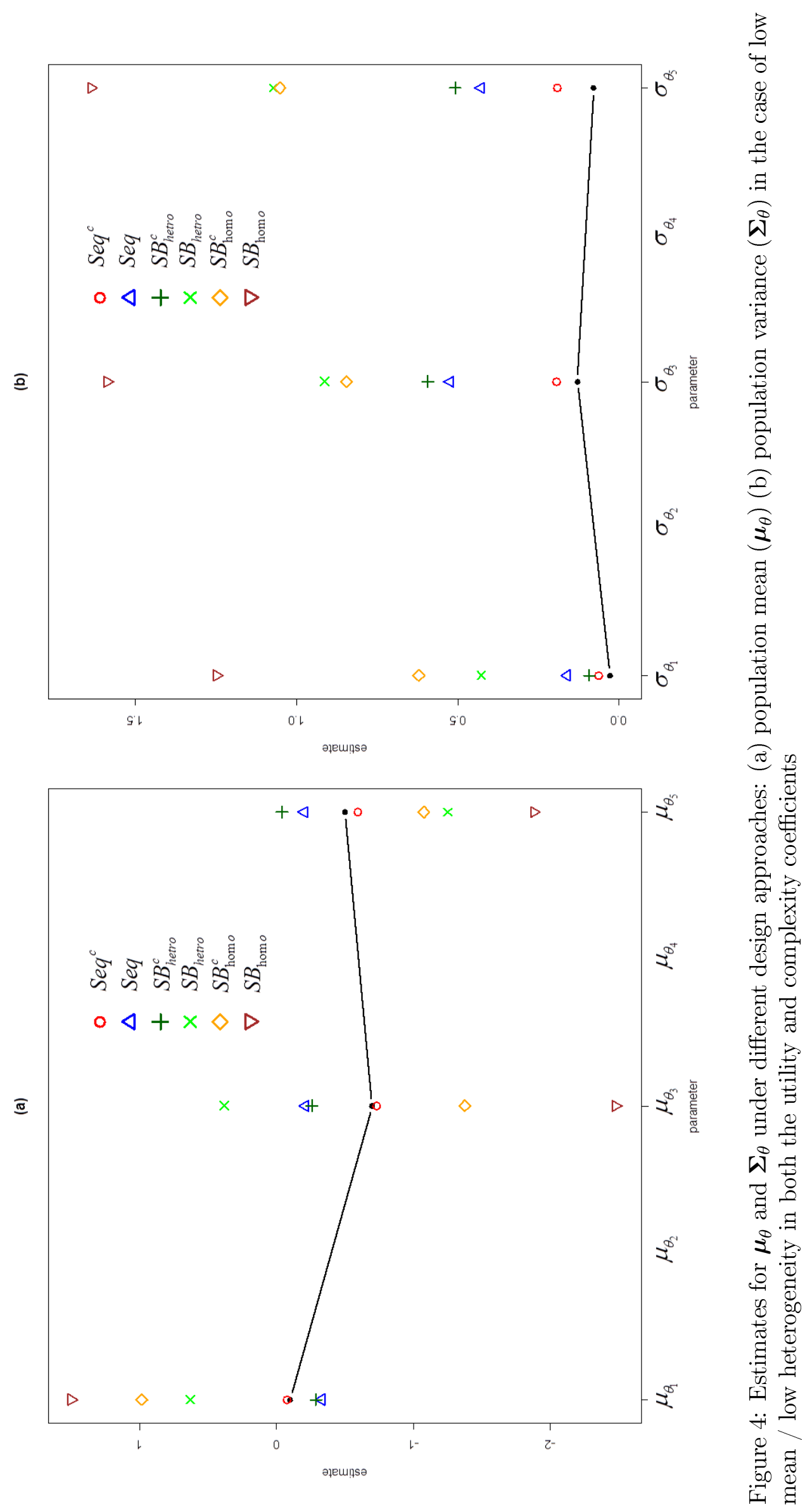



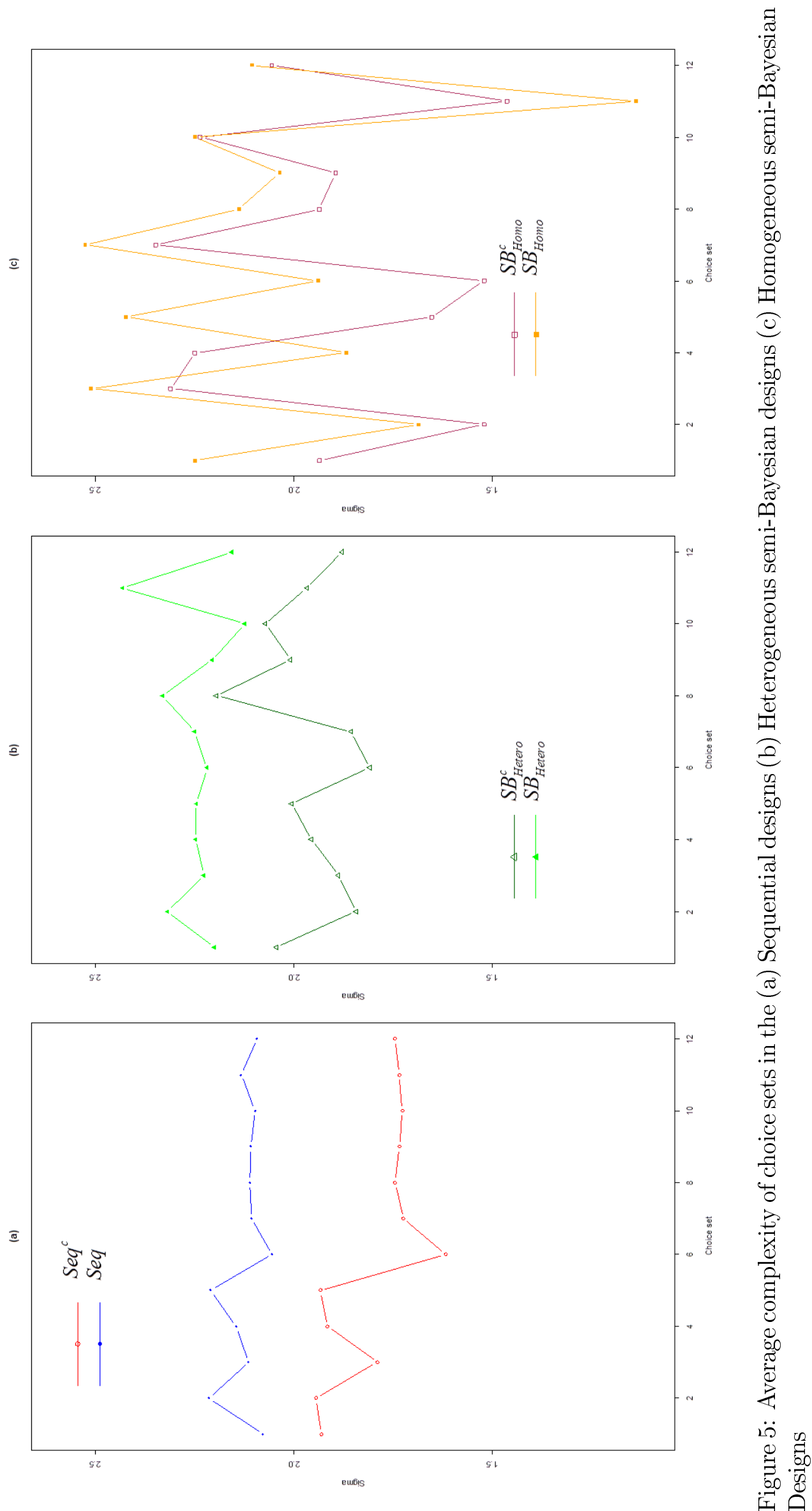
calculated and averaged out over all the respondents. Figure 6 shows the histogram of the average error variance.

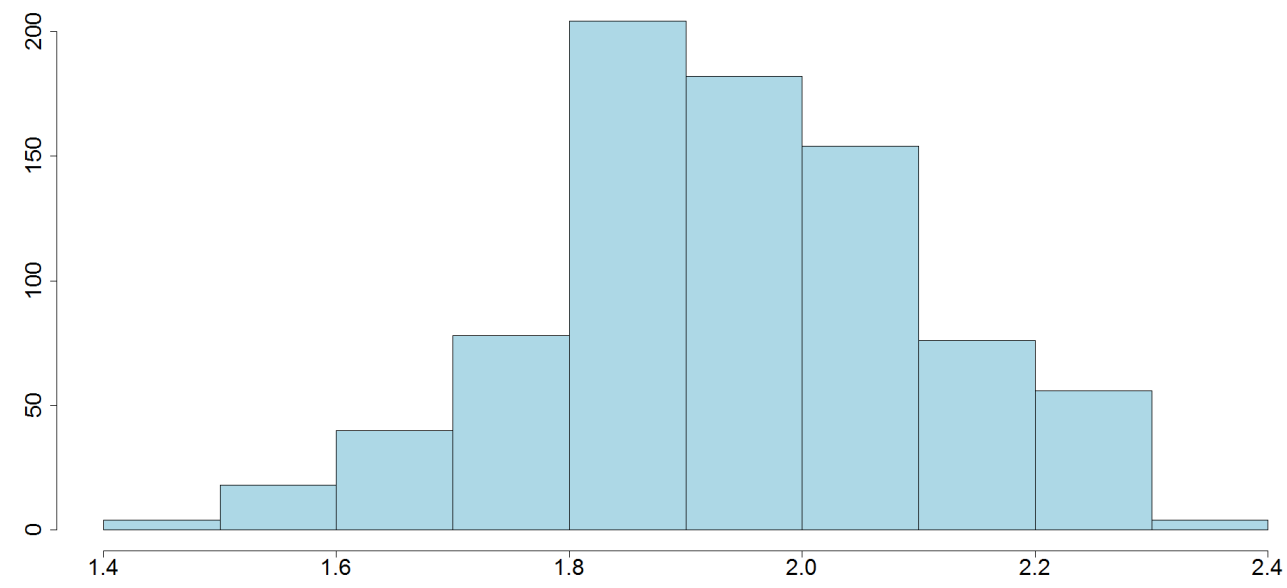

Figure 6: Histogram of average true error variance of all possible choice sets

Figure 5 shows that the sequential design generated taking the choice complexity into account provides choice sets which have an average error variance of 1.7. The histogram in Figure 6 confirms that the sequential approach keeps the error variance at a moderate low level. This is different in the other static designs where the choice sets have different average levels of complexity. The homogeneous semi-Bayesian designs consist of choice sets that are extremely easy or extremely complicated which explains their bad performance.

Apart from the main simulation study presented in this section, we conducted two other studies to generalize and validate the results we obtained. Appendix D presents a simulation study in which we relax the assumption of uncorrelated utility and complexity parameters. The results of this simulation study lead to similar conclusions as presented here. In Appendix E we show that the differences we have found between the designs are indeed significant.

\section{Discussion and conclusions}

The heteroscedastic mixed logit model allows to model both the market heterogeneity and the scale heterogeneity due to choice complexity. In this paper sequential choice designs are developed that take the choice complexity into account at the design stage in order to obtain more informative choice data. We generate sequential designs, heterogeneous semi-Bayesian designs and homogeneous semi-Bayesian designs considering and ignoring the choice complexity. A broad simulation study is conducted to assess the efficiency of each design with respect to the accuracy of the estimated heteroscedastic mixed logit model. 
The simulation results confirm that the sequential designs, especially those that are constructed taking the choice complexity into account, outperform all other designs we considered. It turns out that the sequential design approach selects choice sets that have low complexity which leads to more consistent choice outcomes. This advantage is mainly due to the sequential nature of the approach and to a lesser extent due to the consideration of the choice complexity at the design stage. It is shown that the sequential design, generated by ignoring the complexity, results in choice sets with higher complexity than in the case choice complexity is accounted for in the design stage. The homogeneous semi-Bayesian designs perform badly in all scenarios we looked at. Therefore it seems worthwhile to generate choice sets sequentially and to take the complexity of the generated choice sets into account.

The robustness study showed that the sequential approach is more robust to misspecification of the prior distribution than the static designs. This is due to the sequential nature of the approach. In future research we will investigate how much efficiency is lost if the sequential designs that are generated for the heteroscedastic mixed logit model are used when there are no complexity effects involved. 


\section{Appendix A}

\section{The sequential design approach}

To generate the sequential designs we use the Bayesian D-optimality criterion which is based on the generalized Fisher information matrix (GFIM) (Yu et al. (2012)). The GFIM is obtained by taking the negative expectation of the second derivative of the log-posterior density:

$$
\mathcal{I}_{\text {gfim }}\left(\boldsymbol{\gamma}_{n} \mid \mathbf{x}_{n}\right)=-E\left[\frac{\partial^{2} \log \left(q\left(\boldsymbol{\gamma}_{n} \mid \mathbf{y}_{n}, \mathbf{x}_{n}\right)\right)}{\partial \boldsymbol{\gamma}_{n} \partial \boldsymbol{\gamma}_{n}^{\prime}}\right]
$$

where $\boldsymbol{\gamma}_{n}=\left(\boldsymbol{\beta}_{n}, \boldsymbol{\theta}_{n}\right)$ is a combined parameter vector and $q\left(\boldsymbol{\gamma}_{n} \mid \mathbf{y}_{n}, \mathbf{x}_{n}\right)$ is the individual posterior density corresponding to the individual design $\mathbf{x}_{n}$ and responses $\mathbf{y}_{n}$. This posterior density is proportional to the product of the likelihood and the priors:

$$
q\left(\boldsymbol{\gamma}_{n} \mid \mathbf{y}_{n}, \mathbf{x}_{n}\right) \propto L\left(\mathbf{y}_{n} \mid \mathbf{x}_{n}, \gamma_{n}\right) \phi\left(\boldsymbol{\gamma}_{n} \mid \boldsymbol{\mu}_{\gamma}, \boldsymbol{\Sigma}_{\gamma}\right)
$$

The GFIM can be derived from the Fisher information matrix (FIM) which is the negative expectation of the second derivative of the log-likelihood function. Following Yu et al. (2012), under the multivariate normal prior distribution, the expression (2.12) can be written as:

$$
\mathcal{I}_{\text {gfim }}\left(\gamma_{n} \mid \mathbf{x}_{n}\right)=\mathcal{I}_{\text {fim }}\left(\gamma_{n} \mid \mathbf{x}_{n}\right)+\boldsymbol{\Sigma}_{\gamma}^{-1}
$$

For a given respondent and a choice set, the Fisher information matrix is given by

$$
\mathcal{I}_{f i m}(\boldsymbol{\beta}, \boldsymbol{\theta} \mid \mathbf{x})=e^{\mathbf{c}^{\prime} \boldsymbol{\theta}}\left[\begin{array}{cc}
\mathbf{M}^{\prime} \mathbf{P}^{-1} \mathbf{M} & \mathbf{M}^{\prime} \mathbf{P}^{-1} \mathbf{B} \\
\mathbf{B}^{\prime} \mathbf{P}^{-1} \mathbf{M} & \mathbf{B}^{\prime} \mathbf{P}^{-1} \mathbf{B}
\end{array}\right]
$$

where $\mathbf{c}$ is the vector of complexity measurements of the given choice set, $\mathbf{M}=\left[\mathbf{P}-\mathbf{p} \mathbf{p}^{\prime}\right] \mathbf{x}, \mathbf{B}=$ $\left[\mathbf{P}-\mathbf{p p}^{\prime}\right] \mathbf{x} \boldsymbol{\beta} \mathbf{c}^{\prime}, \mathbf{p}=\left(p_{1}, \ldots, p_{K}\right)$ is the vector of choice probabilities for each of the alternatives in the choice set and $\mathbf{P}=\operatorname{diag}\left(p_{1}, \ldots, p_{K}\right)$. To assess the design efficiency we use the Bayesian D-error which is calculated using the determinant of the inverse of the GFIM. Assuming that $\boldsymbol{\gamma}_{n}=\left(\boldsymbol{\beta}_{n}, \boldsymbol{\theta}_{n}\right)$ follows a multivariate normal distribution with mean $\boldsymbol{\mu}_{\gamma}$ and covariance $\boldsymbol{\Sigma}_{\gamma}$, the Bayesian D-error can be written as

$$
D_{B^{-} \text {error }}=\int\left|\mathcal{I}_{g f i m}\left(\boldsymbol{\beta}_{n}, \boldsymbol{\theta}_{n} \mid \mathbf{x}_{n}\right)\right|^{-\frac{1}{p}} \phi\left(\boldsymbol{\gamma}_{n} \mid \boldsymbol{\mu}_{\gamma}, \boldsymbol{\Sigma}_{\gamma}\right) d \boldsymbol{\gamma}_{n}
$$

The sequential design approach consist of two stages. In the first stage, each respondent is assigned to an initial D-optimal design with $S_{1}$ choice sets of size $K$ generated using a common design prior. For a given respondent $n$, this initial design and the corresponding responses are denoted by $\mathbf{x}_{n}^{S_{1}}$ and $\mathbf{y}_{n}^{S_{1}}$, respectively. The initial choices $\mathbf{y}_{n}^{S_{1}}$ are analysed in a Bayesian way, specifically, by optimizing the $\log$-posterior density $\log q\left(\boldsymbol{\beta}_{n}, \boldsymbol{\theta}_{n} \mid \mathbf{y}_{n}^{S_{1}}, \mathbf{x}_{n}^{S_{1}}\right)$ numerically. In the second stage, the posterior distribution 
obtained from the initial stage is used as the design prior to generate the next choice set $\mathbf{x}_{n}^{S_{1}+1}$. This choice set is chosen by minimizing the Bayesian D-error in expression (16) for the combine design $\left(\mathbf{x}_{n}^{S_{1}}, \mathbf{x}_{n}^{S_{1}+1}\right)$. Once the new choice set is evaluated, the design prior is updated with all $S_{1}+1$ choices and the posterior distribution $q\left(\boldsymbol{\beta}_{n}, \boldsymbol{\theta}_{n} \mid \mathbf{y}_{n}^{S_{1}+1}, \mathbf{x}_{n}^{S_{1}}, \mathbf{x}_{n}^{S_{1}+1}\right)$ is obtained. The updated posterior is used to generate the next choice set. This process is repeated until a pre-specified number of choice sets is attained. The Bayesian modified Fedorov algorithm, also called the profile exchange algorithm, which is introduced by Kessels et al. (2006) is used as the design construction algorithm. 


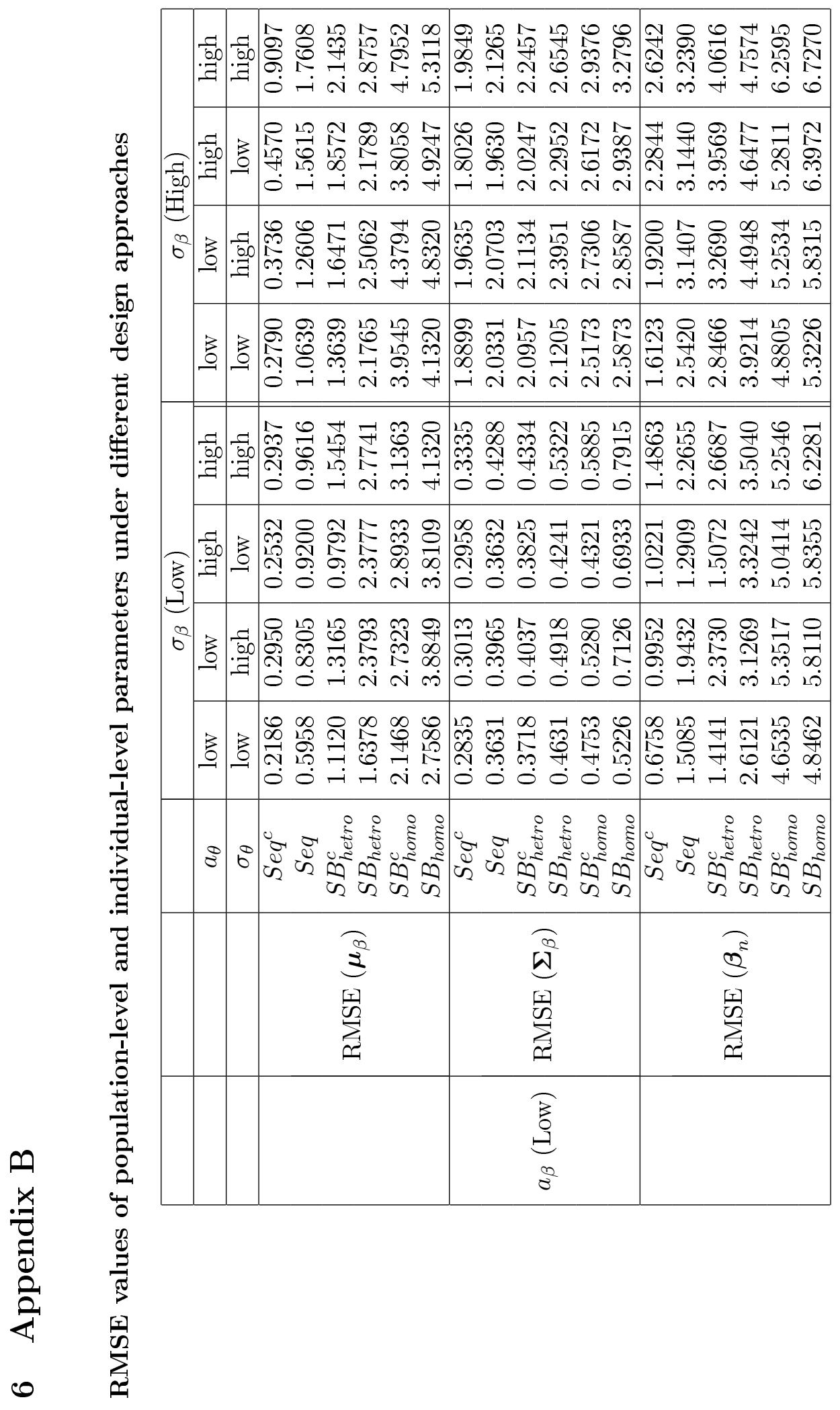




\begin{tabular}{|c|c|c|c|c|c|}
\hline & $\begin{array}{l}-7 \\
.00 \\
\nexists 7\end{array}$ & $\mid \frac{9}{.00}$ & 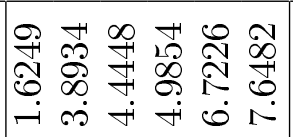 & 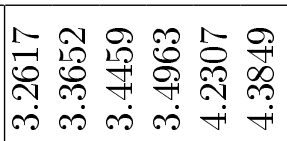 & 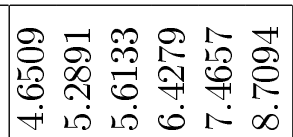 \\
\hline & $\mid \begin{array}{c}-0 \\
\because 00 \\
\exists\end{array}$ & 莕 & 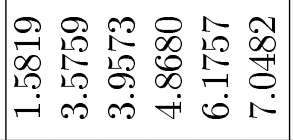 & 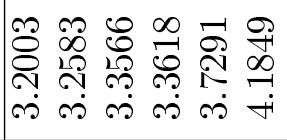 & 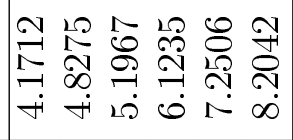 \\
\hline$b$ & 家 & $\mid \begin{array}{c}.0 \\
.00 \\
.\end{array}$ & 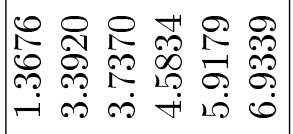 & 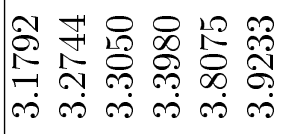 & 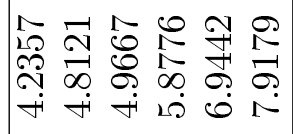 \\
\hline & 音 & 总 & 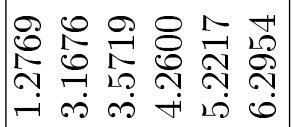 & 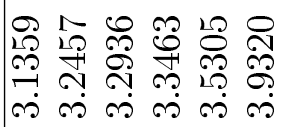 & 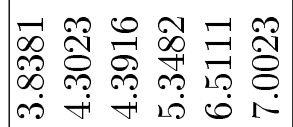 \\
\hline & $\begin{array}{l}.7 \\
.00 \\
\nexists 7\end{array}$ & 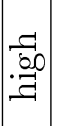 & 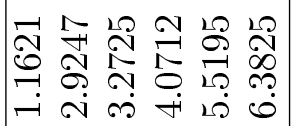 & 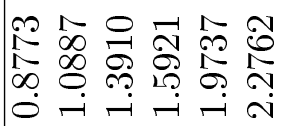 & 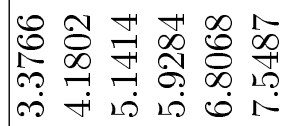 \\
\hline & $\mid \begin{array}{c}-\infty \\
\Xi 0 \\
\Xi\end{array}$ & 㤐 & 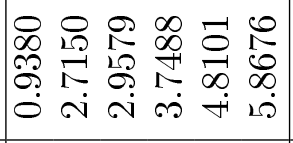 & 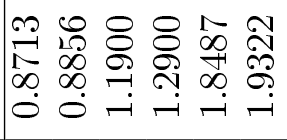 & 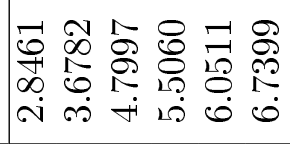 \\
\hline 6 & 2 & 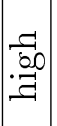 & 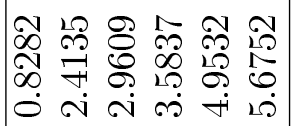 & 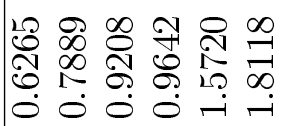 & 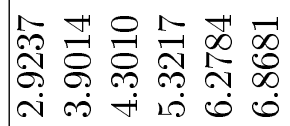 \\
\hline & $\stackrel{B}{0}$ & 莕 & 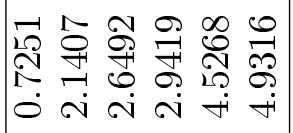 & 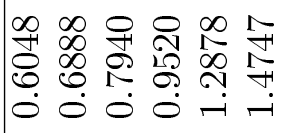 & 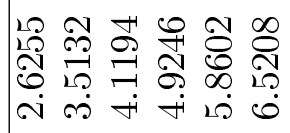 \\
\hline & 8 & 8 & 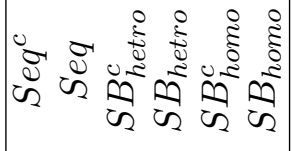 & 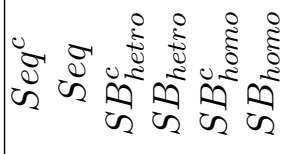 & 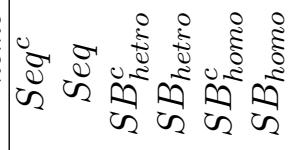 \\
\hline & & & $\sum_{\substack{1 \\
n}}^{\infty}$ & $\underset{\sum_{\infty 1}^{\infty}}{\overbrace{}^{\infty}}$ & 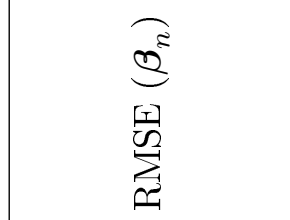 \\
\hline & & & & 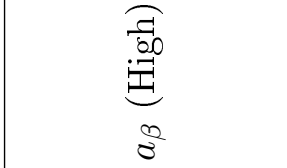 & \\
\hline
\end{tabular}




\section{Appendix C}

\section{Robustness of the designs against misspecified prior distributions}

In the simulation studies we conducted, the assumption that we can use the true heterogeneity distribution as prior in the design stage is not realistic. The true heterogeneity distribution of the model parameters will normally be different from the prior distribution we use to generate the design. We relax this assumption of perfect information and use inference priors that differ from the design prior and assess the estimation accuracy of the utility coefficients. We consider the simulation setting with the full covariance matrix presented in Appendix D and the design prior $\gamma_{n} \sim \mathrm{N}\left(\boldsymbol{\mu}_{\gamma}, \boldsymbol{\Sigma}_{\gamma}\right)$ and the population parameters as defined in Appendix D. We generate choice data based on $\widetilde{\boldsymbol{\gamma}}_{n} \sim \mathrm{N}\left(\widetilde{\boldsymbol{\mu}}_{\gamma}, \widetilde{\boldsymbol{\Sigma}}_{\gamma}\right)$ where $\tilde{\boldsymbol{\mu}}_{\gamma}=\boldsymbol{\mu}_{\gamma}+\delta \mathbf{1}_{6}$ and $\widetilde{\boldsymbol{\Sigma}}_{\gamma}=\alpha \boldsymbol{\Sigma}_{\gamma}$. The parameters $\delta$ and $\alpha$ quantify the degree of deviation of the inference prior from the design prior and set equal to 0.5 and 2. Table 5 gives the results of this robustness study.

Table 5: RMSE values obtained under different design approaches considering different levels of misspecification

\begin{tabular}{|c|c|c|c|c|c|c|}
\hline & $\delta$ & 0 & 0.5 & 0.5 & 2 & 2 \\
\hline & $\alpha$ & 1 & 0.5 & 2 & 0.5 & 2 \\
\hline & $S e q^{c}$ & 1.2031 & 1.2747 & 1.2599 & 1.6374 & 1.5852 \\
\hline & Seq & 1.6699 & 1.7293 & 1.7179 & 1.8239 & 1.9712 \\
\hline & $S B_{\text {hetro }}^{c}$ & 1.5287 & 1.9551 & 1.8606 & 2.3721 & 2.6486 \\
\hline $\boldsymbol{\mu}_{\beta}$ & $S B_{\text {hetro }}$ & 1.8019 & 2.1579 & 1.9313 & 2.4934 & 2.8269 \\
\hline & $S B_{\text {homo }}^{c}$ & 2.1437 & 2.5139 & 2.8160 & 2.6307 & 3.1406 \\
\hline & $S B_{\text {homo }}$ & 2.4931 & 2.8161 & 3.2320 & 3.1422 & 3.4689 \\
\hline & $S e q^{c}$ & 0.5706 & 0.6288 & 0.8275 & 0.9285 & 1.0284 \\
\hline & Seq & 0.6921 & 0.7291 & 0.9085 & 1.0285 & 1.1284 \\
\hline & $S B_{\text {hetro }}^{c}$ & 0.6949 & 0.8291 & 0.9701 & 1.1850 & 1.5282 \\
\hline $\boldsymbol{\Sigma}_{\beta}$ & $S B_{\text {hetro }}$ & 0.7259 & 0.9291 & 1.0862 & 1.2848 & 1.7851 \\
\hline & $S B_{\text {homo }}^{c}$ & 0.8611 & 1.2871 & 1.6272 & 1.8285 & 2.0430 \\
\hline & $S B_{\text {homo }}$ & 0.9597 & 1.3911 & 1.8292 & 2.1184 & 2.2858 \\
\hline & $S e q^{c}$ & 1.2038 & 1.4591 & 1.3962 & 1.5572 & 1.6838 \\
\hline & Seq & 1.6807 & 1.8239 & 1.9470 & 1.9250 & 2.0702 \\
\hline & $S B_{\text {hetro }}^{c}$ & 1.5588 & 1.9928 & 1.9963 & 2.4731 & 2.7641 \\
\hline $\boldsymbol{\beta}_{n}$ & $S B_{\text {hetro }}$ & 1.7039 & 2.0527 & 2.2828 & 2.5963 & 2.9827 \\
\hline & $S B_{\text {homo }}^{c}$ & 2.1422 & 2.4292 & 2.8665 & 2.9318 & 3.3415 \\
\hline & $S B_{\text {homo }}$ & 2.4984 & 2.9105 & 3.1362 & 3.4299 & 3.7477 \\
\hline
\end{tabular}

Table 5 shows that the sequential design constructed taking the choice complexity into account still outperforms the other designs for all levels of misspecification. These are also most robust to the misspecification of the inference prior compared to other static designs. This is expected since the sequential approach updates the prior information repeatedly over the survey. 


\section{Appendix D}

\section{Simulation study with correlation between utility and complexity parameters}

The simulation study we present here considers a full covariance matrix that allows correlations between preference and complexity coefficients. We generate designs with three continuous attributes, three alternatives per choice set and nine choice sets and therefore in this simulation study we estimate three utility coefficients and three complexity coefficients. We assume that the combined parameter vector $\boldsymbol{\gamma}_{n}=\left(\boldsymbol{\beta}_{n}, \boldsymbol{\theta}_{n}\right)$ follows a multivariate normal distribution, that is $\boldsymbol{\gamma}_{n} \sim \mathrm{N}\left(\boldsymbol{\mu}_{\gamma}, \boldsymbol{\Sigma}_{\gamma}\right)$ where $\boldsymbol{\mu}_{\gamma}=[3.054$, $0.922,0.019,-0.194,-1.062,-1.948]$ and covariance matrix

$$
\boldsymbol{\Sigma}_{\gamma}=\left[\begin{array}{rrrrrr}
0.418 & 0.111 & -0.013 & 0.082 & 0.034 & 0.060 \\
0.111 & 0.051 & -0.001 & 0.025 & 0.010 & 0.005 \\
-0.013 & -0.001 & 0.016 & 0.000 & 0.002 & -0.012 \\
0.082 & 0.025 & 0.000 & 0.146 & -0.135 & -0.047 \\
0.034 & 0.010 & 0.002 & -0.135 & 0.239 & -0.083 \\
0.060 & 0.005 & -0.012 & -0.047 & -0.083 & 0.375
\end{array}\right]
$$

We obtained this mean and covariance matrix based on the Swiss-metro data that we introduced in section 2.2. Six designs presented in Table 3 are constructed. To construct the sequential designs, four initial choice sets are used and five choice sets are generated sequentially. To generate semi-Bayesian designs a common prior $\gamma_{n} \sim \mathrm{N}\left(\boldsymbol{\mu}_{\gamma}, \boldsymbol{\Sigma}_{\gamma}\right)$ is used. Design performance is assessed by the RMSE of population and individual-level parameter estimates. Table 6 presents the RMSE values we obtained from this simulation study.

Table 6: RMSE values obtained under different design approaches

\begin{tabular}{ccccccc}
\hline parameter & $S e q^{c}$ & $S e q$ & $S B_{\text {hetro }}^{c}$ & $S B_{\text {hetro }}$ & $S B_{\text {homo }}^{c}$ & $S B_{\text {homo }}$ \\
\hline $\boldsymbol{\mu}_{\beta}$ & 1.2031 & 1.6699 & 1.5287 & 1.8019 & 2.1437 & 2.4931 \\
$\boldsymbol{\Sigma}_{\beta}$ & 0.5706 & 0.6921 & 0.6949 & 0.7259 & 0.8611 & 0.9597 \\
$\boldsymbol{\beta}_{n}$ & 1.2038 & 1.6807 & 1.5588 & 1.7039 & 2.1422 & 2.4984 \\
\hline
\end{tabular}

Results show similar patterns as in the previous study. The sequential design with complexity effects yields the smallest RMSE values for all parameters. The heterogeneous semi-Bayesian design now shows little better performance in estimating the population mean and the individual-level utility coefficients than the sequential design without complexity effects. In estimating the population covariance, these two designs perform equally well. To visualize the estimation accuracy of the preference heterogeneity distribution we generate a beanplot similar to Figure 1.

Figure 7 shows that the sequential approach that takes the complexity into account allows estimation of the true preference heterogeneity distribution more accurately than the other designs. Similar to the 


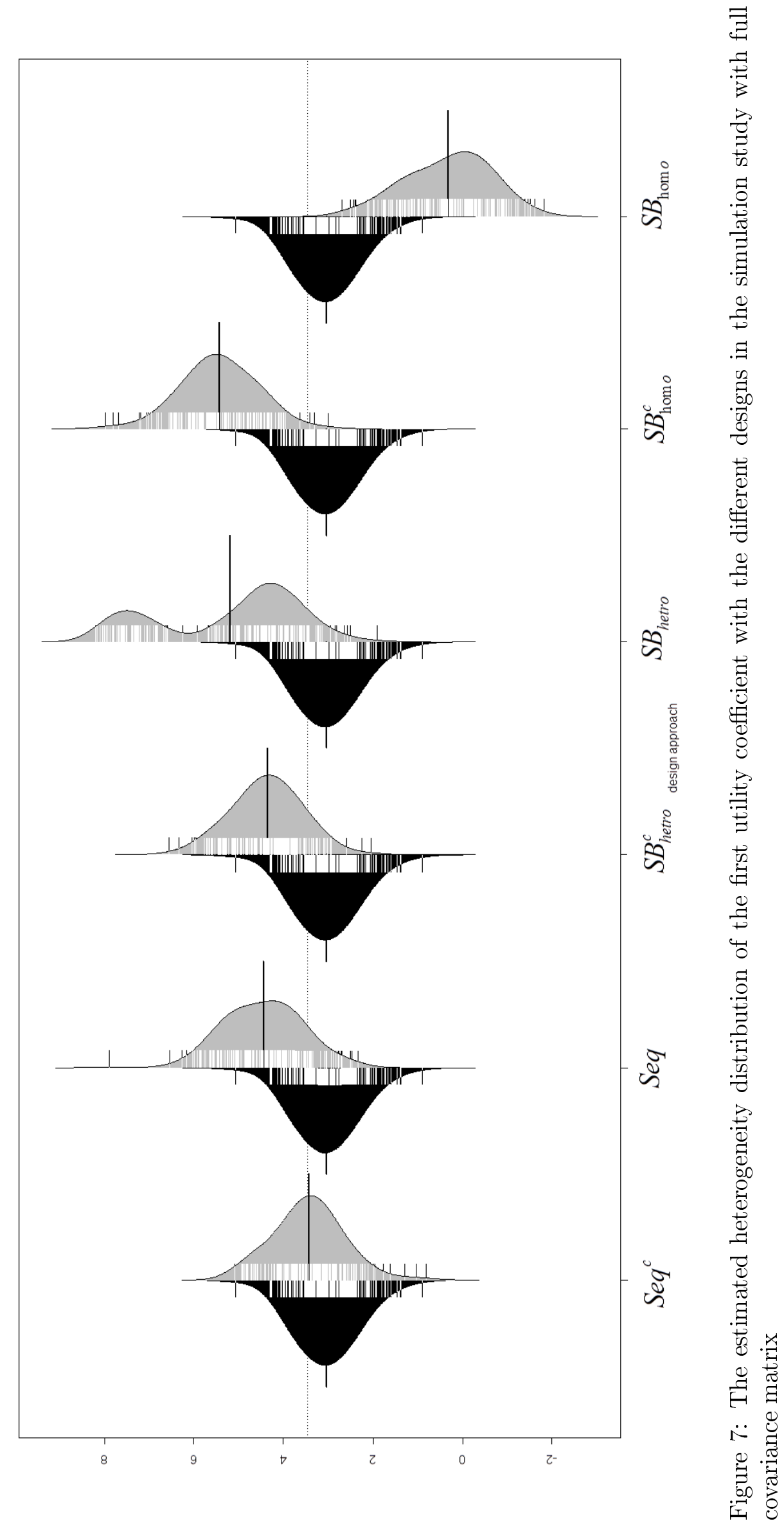


previous results, the homogeneous semi-Bayesian designs show the worst results regardless whether it considers the choice complexity at the design stage or not.

\section{Appendix E}

\section{Validation of the significance of the differences between designs using 100 datasets}

Considering the simulation setting presented in Appendix $\mathrm{C}$ with the full covariance matrix, we simulate 100 data sets and calculate the variance of RMSE for each design approach. Table 6 shows the results. The standard deviations confirm the significance of the results we obtained in the previous simulation studies. As such the conclusions we drew from the above analyses are validated.

Table 7: Means and standard deviations of RMSE

\begin{tabular}{|l|l|l|c|}
\hline & Design & Mean & Std \\
\hline \multirow{5}{*}{$\boldsymbol{\mu}_{\beta}$} & Seq & 1.2151 & 0.0585 \\
& Seq & 1.7107 & 0.0651 \\
& $S B_{\text {hetro }}^{c}$ & 1.6828 & 0.0699 \\
& $S B_{\text {hetro }}$ & 1.7951 & 0.0703 \\
& $S B_{\text {homo }}^{c}$ & 2.1533 & 0.0710 \\
& $S B_{\text {homo }}$ & 2.5441 & 0.0716 \\
\hline \multirow{5}{*}{$\boldsymbol{\Sigma}_{\beta}$} & Seq & 0.5624 & 0.0144 \\
& Seq & 0.6818 & 0.0180 \\
& $S B_{\text {hetro }}^{c}$ & 0.6836 & 0.0183 \\
& $S B_{\text {hetro }}$ & 0.7181 & 0.0173 \\
& $S B_{\text {homo }}^{c}$ & 0.8583 & 0.0211 \\
& $S B_{\text {homo }}$ & 0.9618 & 0.0187 \\
\hline \multirow{5}{*}{$\boldsymbol{\beta}_{n}$} & Seq & 1.2058 & 0.0216 \\
& Seq & 1.7832 & 0.0375 \\
& $S B_{\text {hetro }}^{c}$ & 1.6838 & 0.0276 \\
& $S B_{\text {hetro }}$ & 1.8196 & 0.0363 \\
& $S B_{\text {homo }}^{c}$ & 2.1814 & 0.0375 \\
& $S B_{\text {homo }}$ & 2.5805 & 0.0464 \\
\hline
\end{tabular}




\section{References}

Arora, N., Huber, J., 2001. Improving parameter estimates and model prediction by aggregate customization in choice experiments. Journal of Consumer Research 28 (2), 273-283.

Bliemer, M. C., Rose, J. M., 2010. Construction of experimental designs for mixed logit models allowing for correlation across choice observations. Transportation Research Part B: Methodological 44 (6), $720-734$.

Danthurebandara, V., Yu, J., Vandebroek, M., 2011a. Sequential choice designs to estimate the heterogeneity distribution of willingness-to-pay. Quantitative Marketing and Economics 9, 429-448.

Danthurebandara, V. M., Yu, J., Vandebroek, M., 2011b. Effect of choice complexity on design efficiency in conjoint choice experiments. Journal of Statistical Planning and Inference 141 (7), 2276 2286.

Dellaert, B. G., Donkers, B., Soest, A. V., 2012. Complexity effects in choice experiment-based models. Journal of Marketing Research In press.

Dellaert, B. G. C., Brazell, J. D., Louviere, J. J., 1999. The effect of attribute variation on consumer choice consistency. Marketing Letters 10 (2), 139-147.

DeShazo, J., Fermo, G., 2002. Designing choice sets for stated preference methods: The effects of complexity on choice consistency. Journal of Environmental Economics and Management 44 (1), 123 $-143$.

Kampstra, P., 2008. Beanplot: A boxplot alternative for visual comparison of distributions. Journal of Statistical Software, Code Snippets 28 (1), 1-9.

Keller, K. L., Staelin, R., 1987. Effects of quality and quantity of information on decision effectiveness. Journal of Consumer Research 14 (2), 200-213.

Kessels, R., Goos, P., Vandebroek, M., 2006. A comparison of criteria to design efficient choice experiments. Journal of Marketing Research 43 (3), 409-419.

Mazzotta, M. J., Opaluch, J. J., 1995. Decision making when choices are complex: A test of heiner's hypothesis. Land Economics 71 (4), 500-515.

Palma, A. d., Myers, G. M., Papageorgiou, Y. Y., 1994. Rational choice under an imperfect ability to choose. The American Economic Review 84 (3), 419-440.

Payne, J. W., Bettman, J. R., Johnson, E. J., 1988. Adaptive strategy selection in decision making. Journal of Experimental Psychology: Learning, Memory, and Cognition 14 (3), 534-552.

Sándor, Z., Franses, P., 2009. Consumer price evaluations through choice experiments. Journal of Applied Econometrics 24 (3), 517-535. 
Sándor, Z., Wedel, M., 2005. Heterogeneous conjoint choice designs. Journal of Marketing Research $42(2), 210-218$.

Swait, J., Adamowicz, W., 2001. Choice environment, market complexity, and consumer behavior: A theoretical and empirical approach for incorporating decision complexity into models of consumer choice. Organizational Behavior and Human Decision Processes 86 (2), 141 - 167.

Toubia, O., Hauser, J. R., Simester, D. I., 2004. Polyhedral methods for adaptive choice-based conjoint analysis. Journal of Marketing Research 41 (1), 116-131.

Train, K., 2003. Discrete Choice Methods with Simulation. SUNY-Oswego, Department of Economics.

Wilkie, W. L., 1974. Analysis of effects of information load. Journal of Marketing Research 11 (4), $462-466$.

Yu, J., Goos, P., Vandebroek, M., 2008. Model-robust design of conjoint choice experiments. Communications in Statistics - Simulation and Computation 37 (8), 1603-1621.

Yu, J., Goos, P., Vandebroek, M., 2011. Individually adapted sequential bayesian conjoint-choice designs in the presence of consumer heterogeneity. International Journal of Research in Marketing $28(4), 378-388$.

Yu, J., Goos, P., Vandebroek, M., 2012. A comparison of different bayesian design criteria for setting up stated preference studies. Transportation Research Part B: Methodological (0), -. 